\title{
FINITE DIFFERENCE SCHEMES FOR AN AXISYMMETRIC NONLINEAR HEAT EQUATION WITH BLOW-UP*
}

\author{
CHIEN-HONG $\mathrm{CHO}^{\dagger}$ AND HISASHI OKAMOTO $\ddagger$
}

\begin{abstract}
We study finite difference schemes for axisymmetric blow-up solutions of a nonlinear heat equation in higher spatial dimensions. The phenomenology of blow-up in higher-dimensional space is much more complex than that in one space dimension. To obtain a more complete picture for such phenomena from computational results, it is useful to know the technical details of the numerical schemes for higher spatial dimensions. Since first-order differentiation appears in the differential equation, we pay special attention to it. A sufficient condition for stability is derived. In addition to the convergence of the numerical blow-up time, certain blow-up behaviors, such as blow-up sets and blow-up in the $L^{p}$-norm, are taken into consideration. It is sometimes experienced that a certain property of solutions of a partial differential equation may be lost by a faithfully constructed convergent numerical scheme. The phenomenon of one-point blow-up is a typical example in the numerical analysis of blow-up problems. We prove that our scheme can preserve such a property. It is also remarkable that the $L^{p}$-norm $(1 \leq p<\infty)$ of the solution of the nonlinear heat equation may blow up simultaneously with the $L^{\infty}$-norm or remains bounded in $[0, T)$, where $T$ denotes the blow-up time of the $L^{\infty}$-norm. We propose a systematic way to compute numerical evidence of the $L^{p}$-norm blow-up. The computational results are also analyzed. Moreover, we prove an abstract theorem which shows the relationship between the numerical $L^{p}$-norm blow-up and the exact $L^{p}$-norm blow-up. Numerical examples for higher-dimensional blow-up solutions are presented and discussed.
\end{abstract}

Key words. blow-up, finite difference method, nonlinear heat equation, $L^{p}$-norm blow-up

AMS subject classifications. 65M06, 65M12

1. Introduction. Let $\Omega$ be the unit ball in $\mathbb{R}^{N}$ with the origin as its center. We consider in $\Omega$ the axisymmetric solutions of a nonlinear heat equation of Fujita type, i.e.,

$$
u_{t}=\triangle u+u^{1+\alpha}
$$

where $\triangle$ denotes the Laplace operator and $\alpha>0$ is a given constant. We consider only Dirichlet boundary conditions in the present paper. Since the solution is assumed to be axisymmetric, our target is the following initial and boundary value problem:

$$
\begin{aligned}
u_{t} & =u_{r r}+\frac{N-1}{r} u_{r}+u^{1+\alpha} & & (0 \leq r<1,0<t), \\
u_{r} & =0 & & (r=0), \\
u & =0 & & (r=1), \\
u(0, r) & =u_{0}(r) & & (0 \leq r \leq 1) .
\end{aligned}
$$

Here, we suppose that the initial data $u_{0}(r)$ are continuous in $[0,1], u_{0}(r) \geq 0$ everywhere, and $u_{0}(1)=0$. It was proved (see [5, 14]) that the solutions of (1.2)-(1.5) may become infinite in finite time. This phenomenon is called blow-up, and the time at which the solution becomes infinite is called the blow-up time.

Friedman \& McLeod [14] and Mueller \& Weissler [25] proved that if the initial data $u_{0}$ are monotonically decreasing in $r$, then the solution blows up only at $r=0$. Later, Chen [5] proved a similar proposition for a more general boundary condition with a milder assumption. Numerical experiments tell us that a blow-up in general occurs at a single point, no matter

* Received January 13, 2020. Accepted July 7, 2020. Published online on August 25, 2020. Recommended by Bill Layton.

${ }^{\dagger}$ Department of Mathematics and Advanced Institute of Manufacturing with High-tech Innovations, National Chung Cheng University, Chia-Yi, 621, Taiwan, R.O.C. (chcho2 0 @ ccu . edu . tw).

${ }^{\ddagger}$ Department of Mathematics, Gakushuin University, Tokyo, 171-8588, Japan. 
where the blow-up occurs. But proving this statement is rather difficult. In this regard, [7] is worthy of notice since it proves that the blow-up set consists of finite points. However, this result seems to be proved only in one dimension, namely, only when $N=1$.

It is also remarkable that the solutions of (1.2)-(1.5) may become infinite in a finite time $T$ in the sense that

$$
\|u(t, \cdot)\|_{L^{\infty}} \rightarrow \infty \quad \text { as } \quad t \rightarrow T,
$$

while the $L^{p}$-norms $(1 \leq p<\infty)$ may either become infinite as $t \rightarrow T$ or remain bounded in $[0, T)$. In fact, Friedman \& McLeod [14] showed that

$$
\begin{array}{ll}
\liminf _{t \rightarrow T}\|u(t, \cdot)\|_{L^{p}}=\infty & \text { if } \quad p>\frac{N \alpha}{2}, \\
\limsup _{t \rightarrow T}\|u(t, \cdot)\|_{L^{p}}<\infty & \text { if } \quad p<\frac{N \alpha}{2} .
\end{array}
$$

Weissler [30] showed that (1.6) can be extended to the critical case $p=\frac{N \alpha}{2}$ under some restrictions on the initial data $u_{0}(x)$.

We would like to examine similar problems in a finite difference scheme. Specifically, we would like to construct a finite difference scheme that reproduces the blow-up characteristics described above. It is sometimes experienced that a certain property of solutions of a partial differential equation may be lost by a faithfully constructed convergent numerical solution. Therefore, we consider it important to derive a numerical scheme that preserves such a property. We assume that $N \geq 2$. In the case of $N=1$, we proposed in $[8,11]$ a set of fairly general schemes for computing blow-up solutions. The purpose of the present paper is to compute blow-up solutions in higher dimensions. In two respects this extension is not straightforward. First, the phenomenology of blow-up in higher dimensions is considerably more complex than that in one dimension. In fact, if we consider a higher-dimensional space, then many curious or bizarre phenomena are known to exist. One of them is the recovery of smoothness after a blow-up, which is prohibited in low dimensions, but is known to occur if the spatial dimension is large enough. See [13, 22, 23, 24]. So far we know no successful numerical treatment for reproducing such phenomena. To reproduce them, we believe that we should know the technical details of the numerical schemes for higher dimensions, which were not treated in $[8,9,10,11,12]$. This is one of our motivations for the present paper. Secondly, in computing axisymmetric solutions, we must deal with a term involving first-order derivatives. We need special care for the term in a finite difference scheme.

The number of blow-up points of numerical solutions of (1.2)-(1.5) was studied by Chen [6]. He assumed monotonicity of the initial data $u_{0}(r)$, in which case one-point blow-up is known to occur in (1.2). Nevertheless, he proved that the solution of a finite difference scheme may blow up at more than one point. A similar phenomenon was also proved for a numerical scheme of (1.1) with zero Dirichlet boundary conditions by Groisman [17]. Both of their results suggest that it is difficult for a scheme with a uniform spatial mesh to completely reflect the blow-up phenomena. Besides the number of blow-up points for the numerical solutions, it is worth mentioning that Groisman also proved that the blow-up rate for his numerical solution is completely determined by $\alpha$, while there exist solutions that blow up in different rates for the same nonlinearity $u^{1+\alpha}$ in the continuous problem [19,21]. We will visit this problem in a brief discussion in Section 6.

The rest of this paper is organized as follows. In Section 2, we briefly review two numerical algorithms for the computation of blow-up solutions. In Section 3, we first recall a pioneering work by Chen [6], in which a finite difference scheme was considered for the computation of blow-up solutions of (1.2)-(1.5). Then we propose a class of finite difference 
schemes and derive a sufficient condition for stability. The convergence of the numerical solution is also proved in the same section. In Section 4, we focus on the behavior of the numerical solution, including the numerical blow-up time, the numerical blow-up set, and the $L^{p}$-norm blow-up. In Section 5, we are concerned with the phenomenon of the recovery of smoothness after a blow-up in higher space dimensions. Namely, we try to compute what is called the minimal solution. Finally, the paper ends with concluding remarks.

After having finished the present paper, we learned about the paper [27] by Nakanishi and Saito, where the authors considered blow-up problems of the same kind with the finite element method. In [27], they showed convergence of their scheme and the numerical blow-up time. An analysis of the asymptotic behavior of their numerical solution such as in Section 4 below, however, seems to be out of their scope.

2. Numerical methods for blow-up problems. In this section, we review two algorithms for the computation of blow-up solutions: adaptive and uniform time meshes.

2.1. Adaptive time mesh. Nakagawa [26] considered the one-dimensional semilinear heat equation

$$
\begin{cases}u_{t}=u_{x x}+u^{2} & (0<x<1, t>0), \\ u(0, x)=u_{0}(x) \geq 0 & (0 \leq x \leq 1), \\ u(t, 0)=u(t, 1)=0 & (t>0),\end{cases}
$$

and proposed the following numerical scheme with a uniform spatial mesh:

$$
\left\{\begin{aligned}
\frac{U_{j}^{n+1}-U_{j}^{n}}{\Delta t_{n}} & =\frac{U_{j+1}^{n}-2 U_{j}^{n}+U_{j-1}^{n}}{(\Delta x)^{2}}+\left(U_{j}^{n}\right)^{2} & & (j=1, \ldots, J-1, n \geq 0), \\
U_{j}^{0} & =u_{0}\left(x_{j}\right) & & (j=1, \ldots, J-1), \\
U_{0}^{n} & =U_{J}^{n}=0 & & (n \geq 0),
\end{aligned}\right.
$$

whose temporal grid sizes are defined adaptively by

$$
\Delta t_{n}=\tau \cdot \min \left\{1, \frac{c}{\left\|U^{n}\right\|_{2}}\right\} .
$$

Here, $J \in \mathbb{N}, \Delta x=\frac{1}{J}$ is the spatial grid size, $x_{j}=j \Delta x(0 \leq j \leq J)$ are the spatial grid points, $\tau$ and $c$ are prescribed parameters, and $\lambda=\frac{\tau}{(\Delta x)^{2}} \leq \frac{1}{2}$ is fixed. Moreover, $t_{0}=0$ and $t_{n}=t_{n-1}+\Delta t_{n-1}(n \geq 1)$ denote the temporal grid points, and $U_{j}^{n}$ denotes the approximation of the exact solution $u$ of $(2.1)$ at $\left(t_{n}, x_{j}\right)$. The discrete $L^{p}$-norm is defined by

$$
\left\|U^{n}\right\|_{p}= \begin{cases}\max _{j=1, \ldots, J-1}\left|U_{j}^{n}\right| & \text { if } p=\infty \\ \left(\sum_{j=1}^{J-1} \Delta x\left|U_{j}^{n}\right|^{p}\right)^{\frac{1}{p}} & \text { if } 1 \leq p<\infty\end{cases}
$$

The temporal grid size $\Delta t_{n}$ in (2.2) is defined at each time step according to the discrete $L^{2}$-norm of the numerical solution. As a matter of fact, the discrete $L^{2}$-norm can be replaced by a certain measure of the numerical solution determined a priori; see for instance [11, 17]. A mesh defined in this sense will be called here and hereafter an adaptive mesh. The meaning of (2.2) is that we choose $\Delta t_{n}$ uniformly while $U^{n}$ is not so large, and an adaptive mesh begins to be in effect once $U^{n}$ becomes large. The author in [26] defined the numerical blow-up time as $T(\tau, \Delta x)=\sum_{n=0}^{\infty} \Delta t_{n}$. Then he proved that the numerical solution $U_{j}^{n}$ converges to the 
exact solution $u$ and that the numerical blow-up time $T(\tau, \Delta x)$ is finite and converges to the exact blow-up time of $u$ as $\Delta x \rightarrow 0$.

Nakagawa's method seems to be the first successful attempt to the computation of the blow-up time at which the $L^{\infty}$-norm of the solution becomes infinite. Later, his idea was generalized in [1, 4, 9, 11, 17, 29], in which the asymptotic behavior of the numerical solution was also analyzed. One of our purposes in the present paper is to extend these results to higher dimensions.

2.2. Uniform time mesh. An adaptive temporal grid strategy seems to give a good approximation for blow-up problems, however, we cannot tell from the computational results whether or not the $L^{p}$-norms $(1 \leq p<\infty)$ blow up simultaneously with the $L^{\infty}$-norm. In fact, the unboundedness of the discrete $L^{\infty}$-norm is equivalent to the unboundedness of other discrete $L^{p}$-norms if time-independent spatial grids are applied. To reproduce phenomena such as (1.6), (1.7), a different approach for the computation of blow-up solutions is necessary. For this purpose, we use the idea proposed in [8] and consider finite difference schemes whose temporal grid size is given uniformly $\left(\Delta t_{n}=\Delta t\right.$ for all $\left.n \geq 0\right)$ for the computation of a blow-up in the $L^{p}$-norm.

For convenience, we illustrate the idea given in [8] by the following nonlinear ODE problem

$$
u^{\prime}(t)=G(u), \quad u(0)>0
$$

whose solution blows up at $T_{O} \equiv \int_{u(0)}^{\infty} \frac{d s}{G(s)}$. Here, we assume for simplicity that the nonlinear term $G(u)$ satisfies $G, G^{\prime}, G^{\prime \prime}>0$ and $\int_{u(0)}^{\infty} \frac{d s}{G(s)}<\infty$.

We now consider the forward Euler scheme

$$
\frac{U^{n+1}-U^{n}}{\Delta t}=G\left(U^{n}\right), \quad U^{0}=u(0)
$$

Note that the numerical solutions can be computed for any time $t_{n}=n \Delta t$ even though the exact solution $u$ shows a blown up in finite time $T_{O}$. This implies that our computation should be stopped at a certain finite step. To determine such a step, we impose a nonnegative, strictly increasing function $H$ satisfying $\lim _{s \rightarrow \infty} H(s)=\infty$. One can easily derive by (2.3) and the monotonicity of $G$ that for any given $\Delta t, U^{n}$ is monotonically increasing in $n$ and

$$
U^{n} \rightarrow \infty \quad \text { as } \quad n \rightarrow \infty
$$

Accordingly there should exist a step $n_{\Delta t} \in \mathbb{N}$ depending on $\Delta t$ such that

$$
\Delta t \cdot H\left(U^{n_{\Delta t}-1}\right)<1 \quad \text { and } \quad \Delta t \cdot H\left(U^{n_{\Delta t}}\right) \geq 1 .
$$

We then define $T_{O}(\Delta t)=\Delta t \cdot n_{\Delta t}$, which we call the numerical blow-up time.

THEOREM 2.1. It holds that

$-\int_{H^{-1}\left(\frac{1}{\Delta t}\right)}^{\infty} \frac{d s}{G(s)} \leq T_{O}(\Delta t)-T_{O} \leq-\int_{H^{-1}\left(\frac{1}{\Delta t}\right)}^{\infty} \frac{d s}{G(s)}+\Delta t\left(1+\log \frac{G\left(H^{-1}\left(\frac{1}{\Delta t}\right)\right)}{G\left(U^{0}\right)}\right)$

where $H^{-1}$ denotes the inverse function of $H$. In particular, we have $\lim _{\Delta t \rightarrow 0} T_{O}(\Delta t)=T_{O}$ if the function $H$ satisfies

$$
\Delta t \cdot \log \left(G\left(H^{-1}\left(\frac{1}{\Delta t}\right)\right)\right) \rightarrow 0 \quad \text { as } \quad \Delta t \rightarrow 0 .
$$


For the proof, see [8].

REMARK 2.2. Although the computation is stopped at a finite step $n_{\Delta t}$ where the numerical solution is still finite, the finite-time blow-up is reproduced in the sense that the numerical solution at $T_{O}(\Delta t)$ becomes unbounded as $\Delta t$ tends to 0 ,

$$
U^{n_{\Delta t}} \geq H^{-1}\left(\frac{1}{\Delta t}\right) \rightarrow \infty \quad \text { as } \quad \Delta t \rightarrow 0
$$

and that the numerical blow-up time $T_{O}(\Delta t)$ converges to the exact blow-up time $T_{O}$ as $\Delta t$ tends to 0 ,

$$
T_{O}(\Delta t) \rightarrow T_{O} \quad \text { as } \quad \Delta t \rightarrow 0
$$

Although we have explained the idea via an ODE for the sake of convenience, it can be applied to PDEs, too, as will be shown in Theorem 4.13 below.

REMARK 2.3. We do not use an adaptive spatial mesh in this paper, not because we are uninterested in it. The reason is that our primary goal is to propose a numerical scheme that is supported by a convergence proof. Adaptive spatial meshes are very effective in numerically resolving the concentration of singularities of a solution of PDEs. There may be many references to such schemes, but we cite here only [28]. However, for those schemes it is difficult to prove their convergence mathematically, as far as we know.

REMARK 2.4. If $G(u)$ is a power of $u$, say $G(u)=u^{l}(l>1)$, then (2.4) can be replaced by

$$
\Delta t \cdot \log \left(H^{-1}\left(\frac{1}{\Delta t}\right)\right) \rightarrow 0 \quad \text { as } \quad \Delta t \rightarrow 0 .
$$

In this case, it is easy to see that $H(s)=s^{q}(\forall q>0)$ satisfies (2.5) and thus can be used to compute an approximate blow-up time.

3. Finite difference scheme. We begin this section by recalling the pioneering work by Chen [6], where he considered a scheme for (1.2)-(1.5) in which the nonlinear term is evaluated explicitly and the diffusion term and the first-order derivative term are discretized implicitly by central differences. His scheme is

$$
\frac{U_{j}^{n+1}-U_{j}^{n}}{\Delta t_{n}}=\frac{U_{j+1}^{n+1}-2 U_{j}^{n+1}+U_{j-1}^{n+1}}{(\Delta r)^{2}}+\frac{N-1}{j \Delta r} \frac{U_{j+1}^{n+1}-U_{j-1}^{n+1}}{2 \Delta r}+\left(U_{j}^{n}\right)^{1+\alpha},
$$

where $U_{j}^{n}$ denotes an approximation for $u\left(t_{n}, j \Delta r\right)$. Here, $\Delta r$ is the spatial grid size, and the temporal increment $\Delta t_{n}$ is defined adaptively by

$$
\Delta t_{n}=\tau \cdot \min \left\{1, \frac{c}{\left\|U^{n}\right\|_{p}^{\alpha}}\right\} \quad(1 \leq p \leq \infty)
$$

where $\tau$ and $c$ are prescribed parameters and $\left\|U^{n}\right\|_{p}$ denotes the discrete $L^{p}$-norm:

$$
\left\|U^{n}\right\|_{p}= \begin{cases}\left(\sum_{j=0}^{J-1} \Delta r((j+1) \Delta r)^{N-1}\left|U_{j}^{n}\right|^{p}\right)^{\frac{1}{p}} & (1 \leq p<\infty), \\ \max _{j=0, \ldots, J}\left|U_{j}^{n}\right| & (p=\infty) .\end{cases}
$$


To discretize the boundary condition $u_{r}(t, 0)=0$, he proceeded as follows: Note that (1.3) implies that

$$
\lim _{r \rightarrow 0} \frac{u_{r}(t, r)}{r}=\lim _{r \rightarrow 0} \frac{u_{r}(t, r)-u_{r}(t, 0)}{r}=u_{r r}(t, 0) .
$$

Accordingly, one may assume the following equation:

$$
\frac{U_{0}^{n+1}-U_{0}^{n}}{\Delta t_{n}}=N \frac{-2 U_{0}^{n+1}+2 U_{1}^{n+1}}{(\Delta r)^{2}}+\left(U_{0}^{n}\right)^{1+\alpha} .
$$

This is nothing but an approximation of axisymmetric solutions of $u_{t}=\Delta u+u^{1+\alpha}$ by the central difference at $r=0$. However, the scheme (3.1), together with (3.4), is unstable if $N>3$, as is proved in [6]. In order to overcome this difficulty, Chen [6] proposed an intermediate scheme between (3.1) and (3.4):

$$
\frac{U_{j}^{n+1}-U_{j}^{n}}{\Delta t_{n}}=N \frac{U_{j+1}^{n+1}-2 U_{j}^{n+1}+U_{j-1}^{n+1}}{(\Delta r)^{2}}+\left(U_{j}^{n}\right)^{1+\alpha} \quad \text { for } 1 \leq j<N_{0}:=\left\lfloor\frac{N+1}{2}\right\rfloor,
$$

and (3.1) for $N_{0} \leq j<J$. Here, $\left\lfloor\frac{N+1}{2}\right\rfloor$ denotes the largest integer $\leq \frac{N+1}{2}$. He proved that his scheme is stable for all $N \geq 2$ and converges in the order $O\left((\Delta r)^{2}\right)$. He also analyzed the numerical blow-up set in the case that $u_{0}(r)$ is nonnegative and decreasing in $r \in[0,1]$. He showed that his numerical solution blows up at not only the maximum point but also the point neighboring to it for $\alpha \leq 1$, while the theoretical result tells us that the solution blows up only at $r=0$, namely, the maximum point. A similar result was also mentioned by Groisman in his very interesting work [17] on (1.1). He discretized the partial differential equation by the so-called method of lines to obtain a system of ordinary differential equations. His spatial discretization may be quite general and grids which are not equidistant may be included. But once the spatial grids are set, they are fixed throughout the time evolution.

Both Chen's and Groisman's papers proved, under certain assumptions, that the maxima of their numerical solutions are not isolated blow-up points in the case of $\alpha \leq 1$. Consequently, the question arises as to whether or not we can construct a convergent scheme that faithfully reproduces the one-point blow-up phenomenon for (1.2)-(1.5).

In view of these observations in the previous studies, we propose in this paper a new scheme by which we can reproduce some of the blow-up properties more faithfully than Chen's scheme.

We divide $[0,1]$ into $J$ equal subintervals. Let $r_{j}=j \Delta r \quad(0 \leq j \leq J)$ be the spatial grid points, where $\Delta r=1 / J$. Set $\left.J_{0}=\max \left\{1,\left\lfloor\frac{N-1}{2}\right\rfloor\right]\right\}$. For $j=0$ and $j \geq J_{0}+1$, we consider an explicit version of Chen's scheme, that is,

$$
\frac{U_{0}^{n+1}-U_{0}^{n}}{\Delta t_{n}}=N \frac{-2 U_{0}^{n}+2 U_{1}^{n}}{(\Delta r)^{2}}+\left(U_{0}^{n}\right)^{1+\alpha}
$$

and

$$
\begin{aligned}
& \frac{U_{j}^{n+1}-U_{j}^{n}}{\Delta t_{n}}=\frac{U_{j+1}^{n}-2 U_{j}^{n}+U_{j-1}^{n}}{(\Delta r)^{2}}+\frac{N-1}{j \Delta r} \frac{U_{j+1}^{n}-U_{j-1}^{n}}{2 \Delta r}+\left(U_{j}^{n}\right)^{1+\alpha}, \\
& J_{0}+1 \leq j \leq J-1 .
\end{aligned}
$$

For $1 \leq j \leq J_{0}$, we introduce a new parameter $\sigma$ and approximate $u_{r}$ in the following way:

$$
\begin{aligned}
\frac{N-1}{r} u_{r}(t, r)= & \frac{N-1}{r}\left(\sigma \frac{u(t, r)-u(t, r-\Delta r)}{\Delta r}+(1-\sigma) \frac{u(t, r+\Delta r)-u(t, r)}{\Delta r}\right) \\
& -\frac{(N-1)(1-2 \sigma) \Delta r}{2 r} u_{r r}(t, r)+O\left((\Delta r)^{2}\right) .
\end{aligned}
$$


Note that, for small $r$,

$$
\frac{u_{r}(t, r)}{r}-u_{r r}(t, r)=O\left(r^{2}\right) .
$$

Thus, we have the following approximation

$$
\begin{gathered}
{\left[1+\frac{(1-2 \sigma) \Delta r}{2 r}\right] \frac{N-1}{r} u_{r}(t, r)} \\
=\frac{N-1}{r}\left[\sigma \frac{u(t, r)-u(t, r-\Delta r)}{\Delta r}+(1-\sigma) \frac{u(t, r+\Delta r)-u(t, r)}{\Delta r}\right] \\
+O\left(r^{2}\right)+O\left((\Delta r)^{2}\right) .
\end{gathered}
$$

Here, use has been made of the fact that $u_{r r r}(t, 0)=0 . \sigma$ is a parameter to be chosen. This observation leads us to consider the following scheme: For $1 \leq j \leq J_{0}$,

$$
\begin{aligned}
\frac{U_{j}^{n+1}-U_{j}^{n}}{\Delta t_{n}}=\frac{U_{j-1}^{n}-2 U_{j}^{n}+U_{j+1}^{n}}{(\Delta r)^{2}} & \\
& +\frac{2 j}{2 j+1-2 \sigma} \frac{N-1}{r_{j}}\left(\sigma \frac{U_{j}^{n}-U_{j-1}^{n}}{\Delta r}+(1-\sigma) \frac{U_{j+1}^{n}-U_{j}^{n}}{\Delta r}\right) \\
& +\left(U_{j}^{n}\right)^{1+\alpha} .
\end{aligned}
$$

Note that the truncation error for the scheme (3.7) is of order $(\Delta r)^{2}$ for all $\sigma$. If $\sigma=1 / 2$, then (3.7) is nothing but (3.6). We would like to know which value of $\sigma$ is suited to approximate the blow-up solutions.

We now set $U_{j}^{0}=u_{0}\left(r_{j}\right) \geq 0$, for $0 \leq j \leq J$, and

$$
U_{J}^{n}=0 \quad(\forall n \geq 0) .
$$

Here $\Delta t_{n}>0$ is the temporal grid size, $t_{0}=0$, and $t_{n}=t_{n-1}+\Delta t_{n}$ are the temporal grid points. The temporal increment can either be defined adaptively by

$$
\Delta t_{n}=\tau \cdot \min \left\{1, \frac{c}{\left\|U^{n}\right\|_{p}^{\gamma}}\right\} \quad(\gamma>0)
$$

or be given uniformly by

$$
\Delta t_{n}=\tau \quad(\forall n \geq 0)
$$

In these equations, $\tau>0$ is a prescribed small parameter, and $\left\|U^{n}\right\|_{p}$ is defined as in (3.2).

REMARK 3.1. For $j=0$, besides (3.5), it is also natural to consider the following candidates

$$
U_{0}^{n}=U_{1}^{n} \quad \text { or } \quad U_{0}^{n}=\left(4 U_{1}^{n}-U_{2}^{n}\right) / 3 .
$$

In any of the cases of (3.5) and (3.11), smoothness is implicitly assumed. Since smoothness is lost at the blow-up point, it is not clear which is the best. We again note that the theoretical results tell us that the solution blows up at and only at $r=0$ if $u_{0}(r)$ is nonnegative and decreasing in $r \in[0,1]$. In view of this, $U_{0}^{n}=U_{1}^{n}$ would not be suitable for our purpose, since it automatically implies blow-ups at two points. We therefore exclude this from our consideration. 
A similar situation also occurs for the second choice in (3.11) since $U_{1}^{n} / U_{0}^{n} \geq 3 / 4(\forall n \geq 0)$ if nonnegative solutions are considered. Accordingly, we use (3.5) in what follows.

REMARK 3.2. Our scheme is not a mere explicit version of Chen's scheme except in the case that $\sigma=1 / 2$ and $N=2$. Nor is it a generalization of the scheme of Chen. It is true that our scheme is more complicated than Chen's. However, our scheme has the merit that, in addition to stability and convergence, more blow-up properties are preserved. See the discussions in Section 4.

Now we investigate the stability for the scheme (3.5)-(3.8). For the sake of convenience, we set $\lambda=\frac{\tau}{(\Delta r)^{2}}$ and $\lambda_{n}=\frac{\Delta t_{n}}{(\Delta r)^{2}}(\forall n \geq 0)$. Note that now (3.5)-(3.7) can be rewritten as

$$
U_{0}^{n+1}=\left(1-2 N \lambda_{n}\right) U_{0}^{n}+2 N \lambda_{n} U_{1}^{n}+\Delta t_{n}\left(U_{0}^{n}\right)^{1+\alpha}
$$

for $1 \leq j \leq J_{0}$,

$$
\begin{aligned}
U_{j}^{n+1}=( & \left.-2 \lambda_{n}-\frac{2(N-1)(1-2 \sigma)}{2 j+1-2 \sigma} \lambda_{n}\right) U_{j}^{n} \\
& +\lambda_{n}\left(1-\frac{2 \sigma(N-1)}{2 j+1-2 \sigma}\right) U_{j-1}^{n}+\lambda_{n}\left(1+\frac{2(1-\sigma)(N-1)}{2 j+1-2 \sigma}\right) U_{j+1}^{n} \\
& +\Delta t_{n}\left(U_{j}^{n}\right)^{1+\alpha}
\end{aligned}
$$

and, for $J_{0}+1 \leq j \leq J-1$,

$$
\begin{aligned}
U_{j}^{n+1}=(1- & \left.2 \lambda_{n}\right) U_{j}^{n}+\lambda_{n}\left(1-\frac{N-1}{2 j}\right) U_{j-1}^{n}+\lambda_{n}\left(1+\frac{N-1}{2 j}\right) U_{j+1}^{n} \\
& +\Delta t_{n}\left(U_{j}^{n}\right)^{1+\alpha} .
\end{aligned}
$$

LEMMA 3.3. Suppose that $u_{0}(r)$ is nonnegative. Let $\lambda \leq \frac{1}{2 N}$ be fixed and $\sigma \leq \frac{3}{2 N}$. Let $\left\{U_{j}^{n}\right\}$ be a solution of (3.5)-(3.8). Then, $U_{j}^{n} \geq 0$, for all $n \geq 0$ and $0 \leq j \leq J$.

Proof. The proof is carried out by induction. Assume that $U_{j}^{n} \geq 0$ for all $0 \leq j \leq J$. For $j=0$ and $J_{0}+1 \leq j \leq J-1, U_{j}^{n+1} \geq 0$ follows directly from (3.12), (3.14), and the assumption $\lambda \leq \frac{1}{2 N}$. In the case $1 \leq j \leq J_{0}$, it suffices to show that the coefficients at the right-hand side of (3.13) are nonnegative. For convenience, we set the coefficients at the right-hand side of (3.13) to

$$
\begin{aligned}
& a_{j}^{n}=1-2 \lambda_{n}-\frac{2(N-1)(1-2 \sigma)}{2 j+1-2 \sigma} \lambda_{n}, \\
& b_{j}^{n}=\lambda_{n}\left(1-\frac{2 \sigma(N-1)}{2 j+1-2 \sigma}\right), \quad c_{j}^{n}=\lambda_{n}\left(1+\frac{2(1-\sigma)(N-1)}{2 j+1-2 \sigma}\right) .
\end{aligned}
$$

Since $\sigma \leq \frac{3}{2 N}$ and $1 \leq j$, it is easy to see that $c_{j}^{n} \geq 0$ for all $n \geq 0$ and $j$. Observe also that $b_{j}^{n}>0$ is obvious if $\sigma<0$ and that for $\sigma \geq 0$, we have

$$
1-\frac{2 \sigma(N-1)}{2 j+1-2 \sigma} \geq 1-\frac{2 \sigma(N-1)}{3-2 \sigma}=\frac{3-2 N \sigma}{3-2 \sigma} \geq 0,
$$

which gives nonnegativity of $b_{j}^{n}$. Now it remains to show the nonnegativity of $a_{j}^{n}$. For $\frac{1}{2} \leq \sigma \leq \frac{3}{2 N}$, we have

$$
\frac{2(N-1)(1-2 \sigma)}{2 j+1-2 \sigma} \leq 0
$$


whence we obtain $a_{j}^{n} \geq 1-2 \lambda_{n} \geq 0$. In the case of $\sigma<\frac{1}{2}$, note that

$$
\frac{2(N-1)(1-2 \sigma)}{2 j+1-2 \sigma}<2(N-1)
$$

which implies

$$
a_{j}^{n}>1-2 \lambda_{n}-2(N-1) \lambda_{n}=1-2 N \lambda_{n} \geq 0 .
$$

Thus, we have proved the nonnegativity of $a_{j}^{n}, b_{j}^{n}, c_{j}^{n}$, and we are done.

LEMMA 3.4. Suppose that $u_{0}(r)$ is nonnegative and monotonically decreasing. Let $\lambda \leq \frac{1}{3 N}$ and $\sigma \leq \frac{3}{2 N}$. Let $\left\{U_{j}^{n}\right\}$ be a solution of (3.5)-(3.8). Then, $U_{j}^{n}$ is monotonically decreasing in $j$. Namely, $U_{j}^{n} \geq U_{j+1}^{n} \geq 0$, for all $n \geq 0$ and $0 \leq j \leq J-1$.

Proof. We prove this by induction. Assume that $U_{j}^{n} \geq U_{j+1}^{n} \geq 0$ for a certain $n$ and all $0 \leq j \leq J-1$. Note that for $j=0$,

$$
\begin{aligned}
U_{0}^{n+1}-U_{1}^{n+1}= & \left(1-2 N \lambda_{n}\right) U_{0}^{n}+2 N \lambda_{n} U_{1}^{n}-\left(a_{1}^{n} U_{1}^{n}+b_{1}^{n} U_{0}^{n}+c_{1}^{n} U_{2}^{n}\right) \\
& +\Delta t_{n}\left[\left(U_{0}^{n}\right)^{1+\alpha}-\left(U_{1}^{n}\right)^{1+\alpha}\right] \\
\geq & \left(1-2 N \lambda_{n}-b_{1}^{n}\right) U_{0}^{n}-\left(a_{1}^{n}+c_{1}^{n}-2 N \lambda_{n}\right) U_{1}^{n} .
\end{aligned}
$$

Since

$$
1-2 N \lambda_{n}-b_{1}^{n}=1-2 N \lambda_{n}-\frac{3-2 N \sigma}{3-2 \sigma} \lambda_{n}
$$

and

$$
a_{1}^{n}+c_{1}^{n}-2 N \lambda_{n}=1-\lambda_{n}+\frac{2(N-1) \sigma}{3-2 \sigma} \lambda_{n}-2 N \lambda_{n}=1-\frac{3-2 N \sigma}{3-2 \sigma} \lambda_{n}-2 N \lambda_{n},
$$

we obtain

$$
U_{0}^{n+1}-U_{1}^{n+1} \geq\left(1-2 N \lambda_{n}-\frac{3-2 N \sigma}{3-2 \sigma} \lambda_{n}\right)\left(U_{0}^{n}-U_{1}^{n}\right) .
$$

The lower bound of the quantity in parenthesis as a function of $\sigma$ is easily verified, and we have

$$
U_{0}^{n+1}-U_{1}^{n+1} \geq\left(1-3 N \lambda_{n}\right)\left(U_{0}^{n}-U_{1}^{n}\right) \geq 0
$$

For $1 \leq j \leq J_{0}-1$,

$$
\begin{aligned}
U_{j}^{n+1}-U_{j+1}^{n+1}= & a_{j}^{n} U_{j}^{n}+b_{j}^{n} U_{j-1}^{n}+c_{j}^{n} U_{j+1}^{n}-\left(a_{j+1}^{n} U_{j+1}^{n}+b_{j+1}^{n} U_{j}^{n}+c_{j+1}^{n} U_{j+2}^{n}\right) \\
& \quad+\Delta t_{n}\left[\left(U_{j}^{n}\right)^{1+\alpha}-\left(U_{j+1}^{n}\right)^{1+\alpha}\right] \\
\geq & \left(a_{j}^{n}+b_{j}^{n}\right) U_{j}^{n}+c_{j}^{n} U_{j+1}^{n}-b_{j+1}^{n} U_{j}^{n}-\left(a_{j+1}^{n}+c_{j+1}^{n}\right) U_{j+1}^{n} \\
= & \left(a_{j}^{n}+b_{j}^{n}-b_{j+1}^{n}\right) U_{j}^{n}-\left(a_{j+1}^{n}+c_{j+1}^{n}-c_{j}^{n}\right) U_{j+1}^{n} \\
= & {\left[1-2 \lambda_{n}-\frac{2(N-1)(1-\sigma)}{2 j+1-2 \sigma} \lambda_{n}+\frac{2(N-1) \sigma}{2(j+1)+1-2 \sigma} \lambda_{n}\right]\left(U_{j}^{n}-U_{j+1}^{n}\right) . }
\end{aligned}
$$

Since, for $\sigma \geq 0$,

$$
\begin{aligned}
1-2 \lambda_{n} & -\frac{2(N-1)(1-\sigma)}{2 j+1-2 \sigma} \lambda_{n}+\frac{2(N-1) \sigma}{2(j+1)+1-2 \sigma} \lambda_{n} \\
& \geq 1-2 \lambda_{n}-\frac{2(N-1)(1-\sigma)}{2 j-1+2(1-\sigma)} \lambda_{n} \geq 1-2 \lambda_{n}-(N-1) \lambda_{n} \geq 1-2 N \lambda_{n} \geq 0
\end{aligned}
$$


and for $\sigma<0$,

$$
\begin{aligned}
1-2 \lambda_{n}-\frac{2(N-1)(1-\sigma)}{2 j+1-2 \sigma} \lambda_{n}+\frac{2(N-1) \sigma}{2(j+1)+1-2 \sigma} \lambda_{n} \\
\quad \geq 1-2 \lambda_{n}-\frac{2(N-1)(1-\sigma)}{2 j+1-2 \sigma} \lambda_{n}+\frac{2(N-1) \sigma}{2 j+1-2 \sigma} \lambda_{n} \\
\quad=1-2 \lambda_{n}-\frac{2(N-1)(1-2 \sigma)}{2 j+1-2 \sigma} \lambda_{n} \\
\quad \geq 1-2 \lambda_{n}-2(N-1) \lambda_{n}=1-2 N \lambda_{n} \geq 0,
\end{aligned}
$$

we have $U_{j}^{n+1} \geq U_{j+1}^{n+1}$ for $1 \leq j \leq J_{0}-1$. For $j=J_{0}$, note that

$$
\begin{gathered}
U_{J_{0}}^{n+1}-U_{J_{0}+1}^{n+1}=\left(a_{J_{0}}^{n} U_{J_{0}}^{n}+b_{J_{0}}^{n} U_{J_{0}-1}^{n}+c_{J_{0}}^{n} U_{J_{0}+1}^{n}\right) \\
-\left[\left(1-2 \lambda_{n}\right) U_{J_{0}+1}^{n}+\lambda_{n}\left(1-\frac{N-1}{2\left(J_{0}+1\right)}\right) U_{J_{0}}^{n}\right. \\
\left.\quad+\lambda_{n}\left(1+\frac{N-1}{2\left(J_{0}+1\right)}\right) U_{J_{0}+2}^{n}\right] \\
\quad+\Delta t_{n}\left[\left(U_{J_{0}}^{n}\right)^{1+\alpha}-\left(U_{J_{0}+1}^{n}\right)^{1+\alpha}\right] \\
\geq\left[a_{J_{0}}^{n}+b_{J_{0}}^{n}-\lambda_{n}\left(1-\frac{N-1}{2\left(J_{0}+1\right)}\right)\right] U_{J_{0}}^{n} \\
\quad-\left[\left(1-2 \lambda_{n}\right)+\lambda_{n}\left(1+\frac{N-1}{2\left(J_{0}+1\right)}\right)-c_{J_{0}}^{n}\right] U_{J_{0}+1}^{n} \\
=\left[1-2 \lambda_{n}-\frac{2(N-1)(1-\sigma)}{2 J_{0}+1-2 \sigma} \lambda_{n}+\frac{N-1}{2\left(J_{0}+1\right)} \lambda_{n}\right]\left(U_{J_{0}}^{n}-U_{J_{0}+1}^{n}\right) .
\end{gathered}
$$

Now $U_{J_{0}}^{n+1} \geq U_{J_{0}+1}^{n+1}$ follows from

$$
\begin{aligned}
1-2 \lambda_{n} & -\frac{2(N-1)(1-\sigma)}{2 J_{0}+1-2 \sigma} \lambda_{n}+\frac{N-1}{2\left(J_{0}+1\right)} \lambda_{n} \\
& \geq 1-2 \lambda_{n}-\frac{2(N-1)(1-\sigma)}{2 J_{0}-1+2(1-\sigma)} \lambda_{n} \geq 1-2 \lambda_{n}-(N-1) \lambda_{n} \geq 1-2 N \lambda_{n} \geq 0 .
\end{aligned}
$$

For $j \geq J_{0}+1$, the monotonicity can easily be proved by a similar argument given in [6]. Now the proof of monotonicity is completed by induction.

COROLlary 3.5. For $\sigma=\frac{3}{2 N}$, instead of $\lambda \leq \frac{1}{3 N}$, it suffices to assume $\lambda \leq \frac{1}{2 N}$ to preserve the monotonicity of the numerical solution by virtue of (3.15). If $0 \leq \sigma<3 /(2 N)$, then it is enough to assume that $\lambda \leq 1 /(2 N+1)$.

We now prove convergence of the approximate solution when the exact solution is smooth. The restriction for $\lambda$ in the preceding lemma and corollary is not used here. It will be used in later sections when we consider the monotonicity of the solutions.

THEOREM 3.6. Let $\lambda \leq \frac{1}{2 N}$ and $\sigma \leq \frac{3}{2 N}$. Let $\left\{U_{j}^{n}\right\}$ be a solution of (3.5)-(3.8) and $u(t, x) \in C^{2,4}([0, T) \times[0,1])$ be a solution of $(1.2)-(1.5)$. Then for any $T_{0}<T$, there exists a positive constant $C$, depending only on $T_{0}$ and the initial data, such that

$$
\max _{0 \leq j \leq J}\left|U_{j}^{n}-u\left(t_{n}, r_{j}\right)\right|<C(\Delta r)^{2},
$$

for $t_{n} \leq T_{0}$. 
Proof. Under the stability conditions $\lambda \leq \frac{1}{2 N}$ and $\sigma \leq \frac{3}{2 N}$, the proof is essentially well known. We give a sketch of it for the sake of convenience. Set $e_{j}^{n}=U_{j}^{n}-u\left(t_{n}, r_{j}\right)$. Note that the truncation errors for (3.5)-(3.7) are of order $(\Delta r)^{2}$, whence we have by (3.12)-(3.14),

$$
\begin{aligned}
e_{0}^{n+1}= & \left(1-2 N \lambda_{n}\right) e_{0}^{n}+2 N \lambda_{n} e_{1}^{n}+\Delta t_{n}\left[\left(U_{0}^{n}\right)^{1+\alpha}-\left(u\left(t_{n}, 0\right)\right)^{1+\alpha}+O(\Delta r)^{2}\right], \\
e_{j}^{n+1}= & \left(1-2 \lambda_{n}-\frac{2(N-1)(1-2 \sigma)}{2 j+1-2 \sigma} \lambda_{n}\right) e_{j}^{n}+\lambda_{n}\left(1-\frac{2 \sigma(N-1)}{2 j+1-2 \sigma}\right) e_{j-1}^{n} \\
& +\lambda_{n}\left(1+\frac{2(1-\sigma)(N-1)}{2 j+1-2 \sigma}\right) e_{j+1}^{n} \\
& +\Delta t_{n}\left[\left(U_{j}^{n}\right)^{1+\alpha}-\left(u\left(t_{n}, r_{j}\right)\right)^{1+\alpha}+O(\Delta r)^{2}\right],
\end{aligned}
$$

for $1 \leq j \leq J_{0}$, and for $J_{0}+1 \leq j \leq J-1$,

$$
\begin{aligned}
e_{j}^{n+1}=( & \left.-2 \lambda_{n}\right) e_{j}^{n}+\lambda_{n}\left(1-\frac{N-1}{2 j}\right) e_{j-1}^{n}+\lambda_{n}\left(1+\frac{N-1}{2 j}\right) e_{j+1}^{n} \\
& +\Delta t_{n}\left[\left(U_{j}^{n}\right)^{1+\alpha}-\left(u\left(t_{n}, r_{j}\right)\right)^{1+\alpha}+O(\Delta r)^{2}\right] .
\end{aligned}
$$

Let $E^{n}=\max _{0 \leq j \leq J}\left|e_{j}^{n}\right|$. Since all the coefficients are nonnegative under the stability assumptions $\lambda \leq \frac{1}{2 N}$ and $\sigma \leq \frac{3}{2 N}$, it follows that

$$
\left|e_{j}^{n+1}\right| \leq E^{n}+\Delta t_{n}(1+\alpha)\left(u\left(t_{n}, r_{j}\right)+\left|E^{n}\right|\right)^{\alpha} E^{n}+\Delta t_{n} O\left((\Delta r)^{2}\right) .
$$

Hence,

$$
E^{n+1} \leq\left(1+\bar{C} \Delta t_{n}\right) E^{n}+\Delta t_{n} O\left((\Delta r)^{2}\right),
$$

as long as $E^{n} \leq 1$, where the constant $\bar{C}$ depends only on $u$ and its derivatives on $\left[0, T_{0}\right] \times[0,1]$. This inequality is then used to show that $E^{n} \leq 1$ for all $n$ if the initial error is small enough. Then it is used once more to conclude that $E^{n}=O\left((\Delta r)^{2}\right)$ for all $t_{n} \leq T_{0}$.

4. Behavior of the numerical solution. In this section, we assume for simplicity that the initial data are nonnegative and monotonically decreasing in $r$. We will give some sufficient conditions by which the numerical solution reproduces the blow-up characteristics.

Let $a \in(0,1)$ be given, and define $W_{j}^{n}$ by

$$
W_{j}^{n}=\delta^{2} U_{j}^{n}+(1-a)\left(U_{j}^{n}\right)^{1+\alpha} \quad(\forall j=0, \ldots, J-1),
$$

where

$$
\begin{aligned}
\delta^{2} U_{0}^{n}= & 2 N \frac{U_{1}^{n}-U_{0}^{n}}{(\Delta r)^{2}}, \\
\delta^{2} U_{j}^{n}= & \frac{U_{j-1}^{n}-2 U_{j}^{n}+U_{j}^{n+1}}{(\Delta r)^{2}} \\
& +\frac{2 j}{2 j+1-2 \sigma} \frac{N-1}{r_{j}}\left(\sigma \frac{U_{j}^{n}-U_{j-1}^{n}}{\Delta r}+(1-\sigma) \frac{U_{j}^{n+1}-U_{j}^{n}}{\Delta r}\right) \\
& \quad\left(1 \leq j \leq J_{0}\right)
\end{aligned}
$$

and

$$
\delta^{2} U_{j}^{n}=\frac{U_{j-1}^{n}-2 U_{j}^{n}+U_{j+1}^{n}}{(\Delta r)^{2}}+\frac{N-1}{r_{j}} \frac{U_{j+1}^{n}-U_{j-1}^{n}}{2 \Delta r} \quad\left(J_{0}+1 \leq j \leq J-1\right) .
$$




\section{ETNA}

Kent State University and

Johann Radon Institute (RICAM)

In the following discussion, in addition to the nonnegativity and the monotonicity, we assume that the initial data satisfy

$$
W_{j}^{0} \geq 0 \quad(0 \leq \forall j \leq J-1) \quad \text { for some } a \in(0,1) .
$$

REMARK 4.1. The assumption $(\mathrm{H})$ is borrowed from [1]. It certainly imposes some restriction on the initial data, but it simplifies the analysis. Note that, by (3.5)-(3.7), $W_{j}^{n}$ can also be written as

$$
W_{j}^{n}=\frac{U_{j}^{n+1}-U_{j}^{n}}{\Delta t_{n}}-a\left(U_{j}^{n}\right)^{1+\alpha} .
$$

In view of (4.2) and the Dirichlet boundary condition (3.8), we thus set $W_{J}^{n}=0(n \geq 0)$ for the sake of convenience.

REMARK 4.2. Since (H) and (4.2) imply that

$$
\frac{U_{j}^{1}-U_{j}^{0}}{\Delta t_{0}} \geq a\left(U_{j}^{0}\right)^{1+\alpha} \geq 0,
$$

it can be regarded as a discrete analogue of

$$
u_{t}(0, r) \geq 0 \quad(0<r<1),
$$

which is a sufficient condition of the finite-time blow-up for the solution of (1.2)-(1.5). See [14] for the details. Since we assume that $a>0$, assumption (H) is stronger than (4.3). However, since $a>0$ can be chosen arbitrarily small, the restriction does not appear so serious. Furthermore, it plays an important role when the blow-up rate of the discrete solution is taken into consideration. As a matter of fact, if assumption $(\mathrm{H})$ is satisfied, then the blow-up of the discrete solution is always of Type I. See $[10,18]$ and also the discussion in Section 6. We however admit that assumption $(\mathrm{H})$ may be a technical one.

LEMMA 4.3. Let $\left\{U_{j}^{n}\right\}$ be the solution of (3.5)-(3.8). Let $\lambda \leq \frac{1}{3 N}$ and $\sigma \leq \frac{3}{2 N}$. Assume that there exists $a \in(0,1)$ such that $(\mathrm{H})$ holds. Then $W_{j}^{n} \geq 0$, for all $j=0, \ldots, J-1$ and $n \geq 0$. In particular, we have

$$
\frac{\left\|U^{n+1}\right\|_{\infty}-\left\|U^{n}\right\|_{\infty}}{\Delta t_{n}}=\frac{U_{0}^{n+1}-U_{0}^{n}}{\Delta t_{n}} \geq a\left(U_{0}^{n}\right)^{1+\alpha} \quad(\forall n \geq 0) .
$$

Proof. We follow the recipe used in [1], in which a one-dimensional discrete semilinear heat equation was considered. We write down the details for the readers' convenience.

The proof is carried out by induction. Assume that $W_{j}^{n} \geq 0$ for all $j=0, \ldots, J-1$. By (4.2) and Lemma 3.3, we have

$$
\frac{U_{j}^{n+1}-U_{j}^{n}}{\Delta t_{n}} \geq a\left(U_{j}^{n}\right)^{1+\alpha} \geq 0
$$

which implies that $U_{j}^{n+1} \geq U_{j}^{n}$ for all $j$. Set $V_{j}^{n}:=\frac{U_{j}^{n+1}-U_{j}^{n}}{\Delta t_{n}}$. We then obtain by (4.1),

$$
\begin{aligned}
\frac{W_{j}^{n+1}-W_{j}^{n}}{\Delta t_{n}} & =\frac{\delta^{2} U_{j}^{n+1}-\delta^{2} U_{j}^{n}}{\Delta t_{n}}+(1-a) \frac{\left(U_{j}^{n+1}\right)^{1+\alpha}-\left(U_{j}^{n}\right)^{1+\alpha}}{\Delta t_{n}} \\
& =\delta^{2} V_{j}^{n}+(1-a) \frac{\left(U_{j}^{n+1}\right)^{1+\alpha}-\left(U_{j}^{n}\right)^{1+\alpha}}{\Delta t_{n}},
\end{aligned}
$$


and, by (4.2), $\delta^{2} W_{j}^{n}=\delta^{2} V_{j}^{n}-a \delta^{2}\left[\left(U_{j}^{n}\right)^{1+\alpha}\right]$. Therefore, we have

$$
\frac{W_{j}^{n+1}-W_{j}^{n}}{\Delta t_{n}}-\delta^{2} W_{j}^{n}=(1-a) \frac{\left(U_{j}^{n+1}\right)^{1+\alpha}-\left(U_{j}^{n}\right)^{1+\alpha}}{\Delta t_{n}}+a \delta^{2}\left[\left(U_{j}^{n}\right)^{1+\alpha}\right]
$$

for all $j=0, \ldots, J-1$. Since $U_{j}^{n+1} \geq U_{j}^{n}$ and $0 \leq U_{j+1}^{n} \leq U_{j}^{n} \quad(\forall j=0, \ldots, J-1)$, we obtain the following four inequalities:

$$
\begin{aligned}
\frac{\left(U_{j}^{n+1}\right)^{1+\alpha}-\left(U_{j}^{n}\right)^{1+\alpha}}{\Delta t_{n}} \geq(1+\alpha)\left(U_{j}^{n}\right)^{\alpha} V_{j}^{n} \quad(0 \leq \forall j \leq J-1), \\
\delta^{2}\left[\left(U_{0}^{n}\right)^{1+\alpha}\right] \geq(1+\alpha)\left(U_{0}^{n}\right)^{\alpha} \delta^{2} U_{0}^{n}, \\
\delta^{2}\left[\left(U_{j}^{n}\right)^{1+\alpha}\right]=\frac{1}{(\Delta r)^{2}}\left[\left(1+\frac{(1-\sigma)(N-1)}{2 j+1-2 \sigma}\right)\left(\left(U_{j+1}^{n}\right)^{1+\alpha}-\left(U_{j}^{n}\right)^{1+\alpha}\right)\right. \\
\left.\quad-\left(1-\frac{\sigma(N-1)}{2 j+1-2 \sigma}\right)\left(\left(U_{j}^{n}\right)^{1+\alpha}-\left(U_{j-1}^{n}\right)^{1+\alpha}\right)\right] \\
\geq(1+\alpha)\left(U_{j}^{n}\right)^{\alpha} \delta^{2} U_{j}^{n} \quad\left(1 \leq \forall j \leq J_{0}\right), \\
\delta^{2}\left[\left(U_{j}^{n}\right)^{1+\alpha}\right]=\frac{1}{(\Delta r)^{2}}\left[\left(1+\frac{N-1}{2 j}\right)\left(\left(U_{j+1}^{n}\right)^{1+\alpha}-\left(U_{j}^{n}\right)^{1+\alpha}\right)\right. \\
\left.\quad-\left(1-\frac{N-1}{2 j}\right)\left(\left(U_{j}^{n}\right)^{1+\alpha}-\left(U_{j-1}^{n}\right)^{1+\alpha}\right)\right] \\
\geq(1+\alpha)\left(U_{j}^{n}\right)^{\alpha} \delta^{2} U_{j}^{n} \quad\left(J_{0}+1 \leq \forall j \leq J-1\right) .
\end{aligned}
$$

These inequalities imply

$$
\begin{aligned}
\frac{W_{j}^{n+1}-W_{j}^{n}}{\Delta t_{n}}-\delta^{2} W_{j}^{n} & \geq(1-a)(1+\alpha)\left(U_{j}^{n}\right)^{\alpha} V_{j}^{n}+a(1+\alpha)\left(U_{j}^{n}\right)^{\alpha} \delta^{2} U_{j}^{n} \\
& =(1+\alpha)\left(U_{j}^{n}\right)^{\alpha} W_{j}^{n} \geq 0
\end{aligned}
$$

for all $j=0, \ldots, J-1$. Then the stability assumptions $\lambda \leq \frac{1}{3 N}$ and $\sigma \leq \frac{3}{2 N}$ prove that $W_{j}^{n+1} \geq 0$. Now (4.4) is a direct consequence of Lemma 3.4 and the fact that $W_{0}^{n} \geq 0$ $(\forall n \geq 0)$.

In the following two sections, we consider (3.5)-(3.8) with the temporal increments (3.9) or (3.10). In addition to the convergence of the numerical blow-up time, we show that each scheme can numerically reproduce a certain blow-up behavior well. For the adaptive one (3.9), we classify the exact numerical blow-up set. On the other hand, we use the uniform one to compute numerical evidence for (1.6) and (1.7).

4.1. Adaptive temporal increment (3.9). In this section, we consider (3.5)-(3.8) in which $\Delta t_{n}$ is given by (3.9). We define the numerical blow-up time by $T(\tau, \Delta r)=\sum_{n=0}^{\infty} \Delta t_{n}$.

THEOREM 4.4. Let $T$ denote the blow-up time for the solution of (1.2)-(1.5), and let $\left\{U_{j}^{n}\right\}$ be the solution of (3.5)-(3.8) in which $\Delta t_{n}$ is given by (3.9). Let $\lambda \leq \frac{1}{3 N}$ and $\sigma \leq \frac{3}{2 N}$. Assume that assumption $(\mathrm{H})$ holds and that $0<\gamma<1+\alpha+\tau^{-1}$. Then we have

$$
T(\tau, \Delta r)<\infty \quad \text { and } \quad \lim _{\Delta r \rightarrow 0} T(\tau, \Delta r)=T .
$$

By virtue of Lemma 4.3, the proof can be carried out in almost the same way as in [11]. For details, we refer the readers to [11]. 
Since we assume that the initial data $u_{0}(r)$ are monotonically decreasing, it follows from Lemma 3.4 that $U_{0}^{n}=\left\|U^{n}\right\|_{\infty}$. Then the results in [5, 14, 25], namely blow-up occurs only at $r=0$, suggest that $U_{j}^{n}$ remain bounded for all $1 \leq j$. We will examine if this is actually the case. The reader will see that the following argument goes parallel to those in $[4,11]$. However, because of the existence of $\sigma$, it will be of some use if we explicitly state the theorems and their proofs.

For simplicity, we put $\gamma=\alpha$ and $p=\infty$ in (3.9) in the following discussion. For other choices of $\gamma$ and $p$, the arguments given in [6] and [9, 12] for the one-dimensional nonlinear heat equations can be applied if suitably modified.

LEMMA 4.5. If $\lambda \leq \frac{1}{3 N}$ and $\sigma \leq \frac{3}{2 N}$, then a blow-up solution $\left\{U_{j}^{n}\right\}$ satisfies

$$
\lim _{n \rightarrow \infty} \frac{\left\|U^{n}\right\|_{\infty}}{\left\|U^{n+1}\right\|_{\infty}}=\frac{1}{1+c \tau} .
$$

Proof. Note that we are concerned with only those $n$ 's for which $U_{0}^{n}=\left\|U^{n}\right\|_{\infty}$ is sufficiently large, whence $\Delta t_{n}=c \tau\left(U_{0}^{n}\right)^{-\alpha}$ and $\lambda_{n}=c \lambda\left(U_{0}^{n}\right)^{-\alpha} \rightarrow 0$ as $n \rightarrow \infty$. Therefore, we have by Lemma 3.4,

$$
\frac{\left\|U^{n+1}\right\|_{\infty}}{\left\|U^{n}\right\|_{\infty}}=\frac{U_{0}^{n+1}}{U_{0}^{n}}=1-2 N \lambda_{n}+2 N \lambda_{n} \frac{U_{1}^{n}}{U_{0}^{n}}+c \tau \rightarrow 1+c \tau \quad \text { as } \quad n \rightarrow \infty .
$$

Note here that $0<U_{1}^{n} / U_{0}^{n} \leq 1$.

The contents of the next lemma is weaker than what we actually wish to obtain. But it subsequently plays an important role.

LEMMA 4.6. If $\lambda \leq \frac{1}{3 N}$ and $\sigma \leq \frac{3}{2 N}$, then $U_{1}^{n} / U_{0}^{n}$ tends to 0 as $n \rightarrow \infty$.

Proof. Note again that we are concerned with only those $n$ 's when $U_{0}^{n}=\left\|U^{n}\right\|_{\infty}$ is sufficiently large, whence $\Delta t_{n}=c \tau\left(U_{0}^{n}\right)^{-\alpha}$. In order to save notational spaces, we set

$$
\sigma_{1}=1-\frac{2 \sigma(N-1)}{3-2 \sigma} \quad \text { and } \quad \sigma_{2}=1+\frac{2(1-\sigma)(N-1)}{3-2 \sigma} .
$$

Then, by (3.12), (3.13), we have

$$
\frac{U_{1}^{n+1}}{U_{0}^{n+1}}=\frac{\left\{1-\left(\sigma_{1}+\sigma_{2}\right) \lambda_{n}\right\} U_{1}^{n}+\lambda_{n} \sigma_{1} U_{0}^{n}+\lambda_{n} \sigma_{2} U_{2}^{n}+\Delta t_{n}\left(U_{1}^{n}\right)^{1+\alpha}}{\left(1-2 N \lambda_{n}\right) U_{0}^{n}+2 N \lambda_{n} U_{1}^{n}+\Delta t_{n}\left(U_{0}^{n}\right)^{1+\alpha}} .
$$

Set $a_{n}=U_{1}^{n} / U_{0}^{n}$. Then we have by monotonicity

$$
\begin{aligned}
a_{n+1} & =\frac{\left\{1-\left(\sigma_{1}+\sigma_{2}\right) \lambda_{n}\right\} a_{n}+\lambda_{n} \sigma_{1}+\lambda_{n} \sigma_{2} \frac{U_{2}^{n}}{U_{0}^{n}}+a_{n}^{1+\alpha} \Delta t_{n}\left(U_{0}^{n}\right)^{\alpha}}{1-2 N \lambda_{n}+2 N \lambda_{n} a_{n}+\Delta t_{n}\left(U_{0}^{n}\right)^{\alpha}} \\
& \leq \frac{a_{n}-\lambda_{n} \sigma_{1} a_{n}+\lambda_{n} \sigma_{1}+a_{n}^{1+\alpha} \Delta t_{n}\left(U_{0}^{n}\right)^{\alpha}}{1-2 N \lambda_{n}+2 N \lambda_{n} a_{n}+\Delta t_{n}\left(U_{0}^{n}\right)^{\alpha}} .
\end{aligned}
$$

Assume that $0 \leq \underline{a}:=\liminf _{n \rightarrow \infty} a_{n}<\limsup _{n \rightarrow \infty} a_{n}=: \bar{a} \leq 1$. Then, for any given $\kappa \in(\underline{a}, \bar{a})$, one may choose a subsequence $\left\{a_{n_{k}}\right\}$ such that

$$
a_{n_{k}}<\kappa \quad \text { and } \quad a_{n_{k}+1} \geq \kappa
$$

By (4.5), we have

$$
\kappa \leq \limsup _{k \rightarrow \infty} a_{n_{k}+1} \leq \frac{1+c \tau \kappa^{\alpha}}{1+c \tau} \kappa<\kappa,
$$


which is a contradiction. This proves the existence of $a=\lim _{n \rightarrow \infty} a_{n} \in[0,1]$. Again by (4.5) we have

$$
a \leq \frac{1+c \tau a^{\alpha}}{1+c \tau} a,
$$

which, together with the fact that $a \in[0,1]$, implies $a=0$ or 1 . Note that we have by (4.5),

$$
\frac{a_{n+1}}{a_{n}} \leq \frac{a_{n}+\lambda_{n} \sigma_{1}\left(1-a_{n}\right)+\left(a_{n}\right)^{1+\alpha} \Delta t_{n}\left(U_{0}^{n}\right)^{\alpha}}{a_{n}-2 N \lambda_{n} a_{n}\left(1-a_{n}\right)+a_{n} \Delta t_{n}\left(U_{0}^{n}\right)^{\alpha}}=: \frac{C_{n}}{D_{n}} .
$$

We now use the following inequality, which is proved in an elementary way:

$$
(1-x) \leq \frac{1}{\alpha}\left(1-x^{\alpha}\right) \quad(0<\alpha<1,0<x<1) .
$$

Then for $0<\alpha<1$ we have

$$
\begin{aligned}
C_{n}-D_{n} & =\left(1-a_{n}\right) \Delta t_{n}\left[(\Delta r)^{-2} \sigma_{1}+2 N a_{n}(\Delta r)^{-2}-\frac{a_{n}-a_{n}^{1+\alpha}}{1-a_{n}}\left(U_{0}^{n}\right)^{\alpha}\right] \\
& \leq\left(1-a_{n}\right) \Delta t_{n}\left[(\Delta r)^{-2}\left(2 N+\sigma_{1}\right)-\alpha a_{n}\left(U_{0}^{n}\right)^{\alpha}\right] .
\end{aligned}
$$

On the other hand, if $1 \leq \alpha$, we have $1-a_{n} \leq 1-\left(a_{n}\right)^{\alpha}$, whence

$$
C_{n}-D_{n} \leq\left(1-a_{n}\right) \Delta t_{n}\left[(\Delta r)^{-2}\left(2 N+\sigma_{1}\right)-a_{n}\left(U_{0}^{n}\right)^{\alpha}\right] .
$$

If $\lim a_{n}=a=1$, then $a_{n}\left(U_{0}^{n}\right)^{\alpha} \rightarrow \infty$ as $n \rightarrow \infty$. Then it follows in either case of (4.7) or (4.8) that $\left\{a_{n}\right\}$ is decreasing for large $n$, which contradicts $a=1$. We therefore have $a=0$. (

COROLLARY 4.7. It holds that

$$
\lim _{n \rightarrow \infty} \frac{a_{n+1}}{a_{n}}= \begin{cases}(1+c \tau)^{-1} & \sigma=\frac{3}{2 N}, \\ (1+c \tau)^{-\min \{\alpha, 1\}} & \sigma<\frac{3}{2 N} .\end{cases}
$$

Proof. We prove the statement in the case that $\sigma=\frac{3}{2 N}$. Note that we have by (3.12), (3.13), and Lemmas 3.3, 3.4,

$$
a_{n+1} \geq \frac{\left(1-\sigma_{2} \lambda_{n}\right) a_{n}+c \tau a_{n}^{1+\alpha}}{1-2 N \lambda_{n}+2 N \lambda_{n} a_{n}+c \tau},
$$

which implies

$$
\frac{a_{n+1}}{a_{n}} \geq \frac{1-\sigma_{2} \lambda_{n}+c \tau a_{n}^{\alpha}}{1-2 N \lambda_{n}+2 N \lambda_{n} a_{n}+c \tau} .
$$

Therefore we have $\liminf a_{n+1} / a_{n} \geq 1 /(1+c \tau)$. On the other hand, the upper bound $\lim \sup a_{n+1} / a_{n} \leq 1 /(1+c \tau)$ is easily derived from (4.6).

The proof in the case of $\sigma<\frac{3}{2 N}$ is rather complicated. However, it can be carried out in a way similar to that in Corollary 4.2 of [11] or Theorem 1 of [4], and we may omit it.

We now state and prove the asymptotic behavior in two cases separately:

THEOREM 4.8. Let $\left\{U_{j}^{n}\right\}$ be a blow-up solution of (3.5)-(3.8), and let $\lambda \leq \frac{1}{2 N}$ and $\sigma=\frac{3}{2 N}$. Then, for all $\alpha>0$, we have $U_{0}^{n} \rightarrow \infty$ as $n \rightarrow \infty$, while $U_{j}^{n}$ remain bounded for all $1 \leq j \leq J$. 
Proof. Since $U_{0}^{n}=\max _{j} U_{j}^{n}$, the assumption that $\left\{U_{j}^{n}\right\}$ is a blow-up solution verifies the first statement. In order to prove the latter part, it suffices to show the boundedness of $U_{1}^{n}$ since Lemma 3.4 implies that $U_{j}^{n} \leq U_{1}^{n}$, for all $j=2, \ldots, J$. We have by (3.13) and Lemma 3.4,

$$
\begin{aligned}
U_{1}^{n+1} & =\left(1-\sigma_{2} \lambda_{n}\right) U_{1}^{n}+\sigma_{2} \lambda_{n} U_{2}^{n}+\Delta t_{n}\left(U_{1}^{n}\right)^{1+\alpha} \\
& \leq U_{1}^{n}\left(1+\Delta t_{n}\left(U_{1}^{n}\right)^{\alpha}\right)=U_{1}^{n}\left(1+c \tau\left(a_{n}\right)^{\alpha}\right) .
\end{aligned}
$$

Here $\sigma_{2}$ is the same one as that given in the proof of Lemma 4.6. Note that $\sigma_{1}=0$ since $2 N \sigma=3$. Thus, the relation is independent of $U_{0}^{n}$. Repeating this argument, we get to

$$
U_{1}^{n+1} \leq U_{1}^{0} \prod_{k=0}^{n}\left(1+c \tau\left(a_{k}\right)^{\alpha}\right) \leq U_{1}^{0} \exp \left(c \tau \sum_{k=0}^{n}\left(a_{k}\right)^{\alpha}\right)<U_{1}^{0} \exp \left(c \tau \sum_{k=0}^{\infty}\left(a_{k}\right)^{\alpha}\right) .
$$

By Corollary 4.7, we have

$$
\sum_{k=0}^{\infty}\left(a_{k}\right)^{\alpha}<\infty
$$

which implies that $\limsup _{n \rightarrow \infty} U_{1}^{n}<\infty$. Accordingly, $\limsup _{n \rightarrow \infty} U_{j}^{n}<\infty$, for all $j=1, \ldots, J$.

The case of $\sigma<3 /(2 N)$ is dealt with in the next theorem.

THEOREM 4.9. Let $\left\{U_{j}^{n}\right\}$ be a blow-up solution of (3.5)-(3.8), and let $\lambda \leq \frac{1}{3 N}$ and $\sigma<\frac{3}{2 N}$. Then, for $\alpha \leq 1$, not only $U_{0}^{n} \rightarrow \infty$ holds true, but also $U_{1}^{n} \rightarrow \infty$ as $n \rightarrow \infty$. If $\alpha>1$, then $U_{j}^{n}$ are bounded for all $1 \leq j \leq J$.

Proof. By (3.13), we have

$$
U_{1}^{n+1} \geq\left\{1-\left(\sigma_{1}+\sigma_{2}\right) \lambda_{n}\right\} U_{1}^{n}+\lambda_{n} \sigma_{1} U_{0}^{n}=\left\{1-\left(\sigma_{1}+\sigma_{2}\right) \lambda_{n}\right\} U_{1}^{n}+c \lambda \sigma_{1}\left(U_{0}^{n}\right)^{1-\alpha},
$$

which implies, for all $\alpha \leq 1$,

$$
\liminf _{n \rightarrow \infty} U_{1}^{n+1} \geq \liminf _{n \rightarrow \infty} U_{1}^{n}+C_{0}
$$

where $C_{0}=+\infty$ if $\alpha<1$, and $C_{0}=c \lambda \sigma_{1}>0$ if $\alpha=1$. In either case the present theorem follows when $\alpha \leq 1$.

Suppose now that $\alpha>1$. Then (3.13) implies that

$$
U_{1}^{n+1} \leq\left(1+c \tau a_{n}^{\alpha}\right) U_{1}^{n}+c \lambda \sigma_{1}\left(U_{0}^{n}\right)^{1-\alpha} .
$$

We then employ the following inequality which was used in [4] in a form as presented in [11, Lemma 4.7].

LEMMA 4.10. Suppose that a positive sequence $\left\{x_{n}\right\}$ satisfies

$$
x_{n+1} \leq A_{n} x_{n}+B_{n} \quad(n=1,2, \ldots),
$$

where the coefficients $A_{n}$ and $B_{n}$ are positive and satisfy

$$
\prod_{n=1}^{\infty} A_{n}<\infty, \quad \sum_{n=1}^{\infty} B_{n}<\infty
$$

Then the sequence $\left\{x_{n}\right\}$ is bounded. 
We omit the proof since it is elementary and is presented in [11].

Set $A_{n}=1+c \tau a_{n}^{\alpha}$ and $B_{n}=c \lambda \sigma_{1}\left(U_{0}^{n}\right)^{1-\alpha}$. Then if this lemma is applied to (4.9), together with Corollary 4.7 and Lemma 4.5 , the boundedness of $\left\{U_{1}^{n}\right\}$ follows. The monotonicity of $U_{j}^{n}$ in $j$ completes the proof.

REMARK 4.11. Although $U_{1}^{n} \rightarrow \infty$ as $n \rightarrow \infty$ for all $\alpha \leq 1$, we still have

$$
a_{n}=\frac{U_{1}^{n}}{U_{0}^{n}} \rightarrow 0 \quad \text { as } \quad n \rightarrow \infty
$$

as is proved in Lemma 4.6. We may phrase this fact as a one-point blow-up in a weak sense.

It is remarkable that, for the case of $\alpha \leq 1$, the number of blow-up points of the numerical solution increases as $\alpha$ decreases. A similar phenomenon was found by [11] in the case of $N=1$. To see this in the present case, we first observe that, by (3.13),

$$
\begin{aligned}
\frac{U_{1}^{n+1}}{U_{1}^{n}}=( & \left.1-2 \lambda_{n}-\frac{2(N-1)(1-2 \sigma)}{3-2 \sigma} \lambda_{n}\right) \\
& +c \lambda\left(1-\frac{2 \sigma(N-1)}{3-2 \sigma}\right) \frac{\left(U_{0}^{n}\right)^{1-\alpha}}{U_{1}^{n}} \\
& +\lambda_{n}\left(1+\frac{2(1-\sigma)(N-1)}{3-2 \sigma}\right) \frac{U_{2}^{n}}{U_{1}^{n}}+c \tau\left(a_{n}\right)^{\alpha},
\end{aligned}
$$

which, together with Lemma 3.4 and Corollary 4.7, implies

$$
\lim _{n \rightarrow \infty} \frac{\left(U_{0}^{n}\right)^{1-\alpha}}{U_{1}^{n}}=\frac{(1+c \tau)^{1-\alpha}-1}{c \lambda\left(1-\frac{2 \sigma(N-1)}{3-2 \sigma}\right)} .
$$

Since, by (3.13),

$$
\begin{aligned}
U_{2}^{n+1} \geq( & \left.1-2 \lambda_{n}-\frac{2(N-1)(1-2 \sigma)}{5-2 \sigma} \lambda_{n}\right) U_{2}^{n} \\
& +c \lambda\left(1-\frac{2 \sigma(N-1)}{5-2 \sigma}\right) \frac{U_{1}^{n}}{\left(U_{0}^{n}\right)^{1-\alpha}}\left(U_{0}^{n}\right)^{1-2 \alpha}
\end{aligned}
$$

one has, by (4.10),

$$
\liminf _{n \rightarrow \infty} U_{2}^{n+1} \geq \liminf _{n \rightarrow \infty} U_{2}^{n}+C_{1}
$$

for some $C_{1} \in(0, \infty]$ if $\alpha \leq 1 / 2$. This implies the unboundedness of $U_{2}^{n}$ in the case of $\alpha \leq 1 / 2$. On the other hand, one can show that $U_{2}^{n}$ remains bounded if $\alpha>1 / 2$ in a way similar to the arguments in Theorem 4.9 for the boundedness of $U_{1}^{n}$. That is, the numerical solution blows up at exactly two points $\left\{r_{0}, r_{1}\right\}$ if $\alpha \in(1 / 2,1]$, while the numerical solution blows up at no less than three points $\left\{r_{0}, r_{1}, r_{2}\right\}$ if $\alpha \leq 1 / 2$. In fact, we have the following theorem:

THEOREM 4.12. Let $\left\{U_{j}^{n}\right\}$ be a blow-up solution of (3.5)-(3.8), and let $\lambda \leq \frac{1}{3 N}$ be fixed and $\sigma<\frac{3}{2 N}$. Let $k$ be any positive integer and assume that

$$
\frac{1}{k+1}<\alpha \leq \frac{1}{k}
$$

Then the numerical solution blows up at exactly $k+1$ points $\left\{r_{0}, r_{1}, \ldots, r_{k}\right\}$. That is,

$$
\lim _{n \rightarrow \infty} U_{j}^{n}=\infty \quad \text { if and only if } \quad 0 \leq j \leq k .
$$



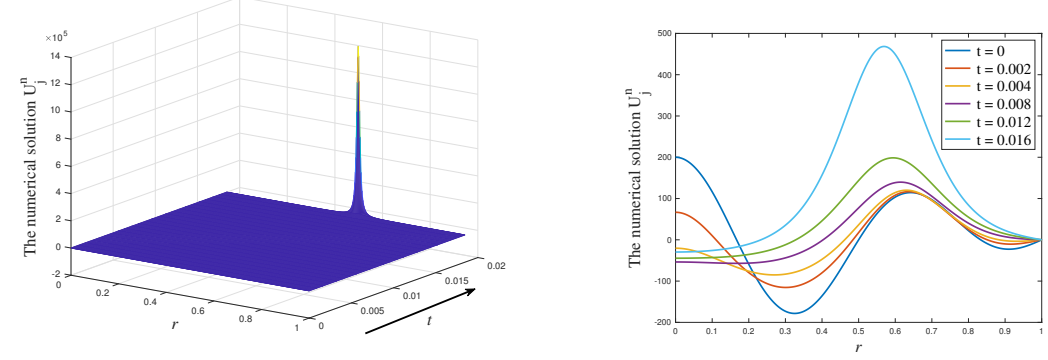

FIG. 4.1. $\quad N=5, \alpha=1, u_{0}(r)=200\left(1-r^{2}\right) \cos (3 \pi r), J=500$, and $\sigma=3 / 10$. The figure at the right-hand side is the graph of the initial data and the profiles of subsequent times.

The proof can be carried out in a way similar to that given in [11], in which a finite difference scheme for a one-dimensional nonlinear heat equation is considered. We thus omit the details.

We have considered those initial data in which blow-up occurs at $r=0$. This is because we can give a rigorous proof in a simple manner. There are also those initial data for which a blow-up occurs at an interior point $r \in(0,1)$. Our scheme can still compute such solutions accurately. Here we present a numerical example with initial data of general shape. In Figure 4.1, the numerical solution shows that blow-up does not always occur at $r=0$ though the maximum of the initial function is attained at $r=0$. This is not surprising since the initial function is neither monotonically decreasing nor nonnegative in $[0,1]$.

4.2. Uniform temporal increment (3.10). In this section, we consider the finite difference solution (3.5)-(3.8) to which (3.10) is applied.

To see how finite-time blow-up is reproduced, we choose a strictly increasing function $H:(0, \infty) \longmapsto(0, \infty)$ satisfying $\lim _{s \rightarrow \infty} H(s)=\infty$. Since we have by Lemma 3.4 and (4.4) that

$$
\begin{aligned}
\left\|U^{n+1}\right\|_{\infty} & =U_{0}^{n+1} \geq\left(1+a \tau\left(U_{0}^{n}\right)^{\alpha}\right) U_{0}^{n} \geq\left(1+a \tau\left(U_{0}^{0}\right)^{\alpha}\right) U_{0}^{n} & \\
& \geq\left(1+a \tau\left(U_{0}^{0}\right)^{\alpha}\right)^{n+1} U_{0}^{0} \rightarrow \infty & \text { as } \quad n \rightarrow \infty,
\end{aligned}
$$

for any given $\tau>0$, there exists a positive integer $n_{\tau}$ such that

$$
\tau \cdot H\left(\left\|U^{n_{\tau}-1}\right\|_{\infty}\right)<1 \quad \text { and } \quad \tau \cdot H\left(\left\|U^{n_{\tau}}\right\|_{\infty}\right) \geq 1 .
$$

We then define the numerical blow-up time $T(\tau)$ by $T(\tau)=\tau \cdot n_{\tau}$. By a similar argument as given in [8], one can prove the following theorem.

THEOREM 4.13. Let $T$ denote the blow-up time for the solution of (1.2)-(1.5), and let $\left\{U_{j}^{n}\right\}$ be the solution of (3.5)-(3.8) in which $\Delta t_{n}$ is given uniformly by (3.10). Let $\lambda \leq \frac{1}{3 N}$ and $\sigma \leq \frac{3}{2 N}$. Assume also that $(\mathrm{H})$ holds. Assume finally that the function $H$ satisfies

$$
\tau \cdot \log \left(H^{-1}\left(\frac{1}{\tau}\right)\right) \rightarrow 0 \quad \text { as } \quad \tau \rightarrow 0
$$

Then we have $\lim _{\tau \rightarrow 0} T(\tau)=T$.

Now we consider the question concerning whether the $L^{p}$-norm of a blow-up solution blows up at the blow-up time $T$ or remains bounded in $t \in[0, T)$. Our algorithm is as follows (see also [10, 18]): 


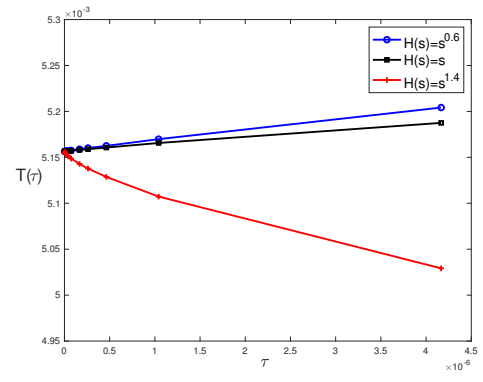
$s, s^{1.4}$.

FIG. 4.2. $N=3, \alpha=1, u_{0}(r)=200\left(1-r^{2}\right)$. Numerical blow-up time $T(\tau)$ computed by $H(s)=s^{0.6}$,

1. Choose a suitable $H$ satisfying (4.12) and compute the numerical blow-up time $T(\tau)=\tau \cdot n_{\tau}$.

2. Compute the discrete $L^{p}$-norm at the time step $n_{\tau}$.

3. Let $\tau \rightarrow 0$ to see whether the discrete $L^{p}$-norms tend to infinity or remain bounded.

In the following computation, we set $N=3, \alpha=1, \sigma=\frac{3}{2 N}=\frac{1}{2}$, and $u_{0}(r)=$ $200\left(1-r^{2}\right)(0<r<1)$. Figure 4.2 illustrates the numerical blow-up times computed by $H(s)=s^{0.6}, s, s^{1.4}$. It seems that the computational result for $H(s)=s$ gives an estimate for the blow-up time better than the others. In fact, since we have by Lemmas 3.4 and 4.3 and assumption $(\mathrm{H})$ that there exists $a \in(0,1)$ such that

$$
a\left(U_{0}^{n}\right)^{1+\alpha} \leq \frac{U_{0}^{n+1}-U_{0}^{n}}{\tau} \leq\left(U_{0}^{n}\right)^{1+\alpha}
$$

the asymptotic behavior of $\left\|U^{n}\right\|_{\infty}=U_{0}^{n}$ might be similar to that of the finite difference solution

$$
\frac{Z^{n+1}-Z^{n}}{\tau}=c\left(Z^{n}\right)^{1+\alpha}, \quad Z^{0}>0
$$

where $c$ is a constant. As a result, from the discussion given in $[8,10], H(s)=s^{\alpha}$ might perhaps be a good choice for the computation of blow-up solutions of (4.13), and thus for the computation of blow-up solutions of (1.2)-(1.5).

Consequently, we set $H(s)=s$ to compute the discrete $L^{p}$-norms at the numerical blow-up time $T(\tau)=\tau \cdot n_{\tau}$. In our settings, (1.6) and (1.7) give

$$
\limsup _{t \rightarrow T}\|u(t, \cdot)\|_{L^{1}}<\infty \quad \text { and } \quad \liminf _{t \rightarrow T}\|u(t, \cdot)\|_{L^{2}}=\infty
$$

Our computational results suggest that the numerical solution behaves in the same way as the exact solution; see Figure 4.3. As a matter of fact, we have the following theorem:

THEOREM 4.14. Set $H(s)=s^{q}(q>0)$. Then, for all $p>\frac{N q}{2}$, it holds that

$$
\lim _{\tau \rightarrow 0}\left\|U^{n_{\tau}}\right\|_{p}=\infty
$$

Proof. By definition (3.2), we have

$$
\left\|U^{n_{\tau}}\right\|_{p} \geq(\Delta r)^{\frac{N}{p}} U_{0}^{n_{\tau}}=(\Delta r)^{\frac{N}{p}}\left\|U^{n_{\tau}}\right\|_{\infty} \geq(\Delta r)^{\frac{N}{p}}\left(\frac{1}{\tau}\right)^{\frac{1}{q}}
$$



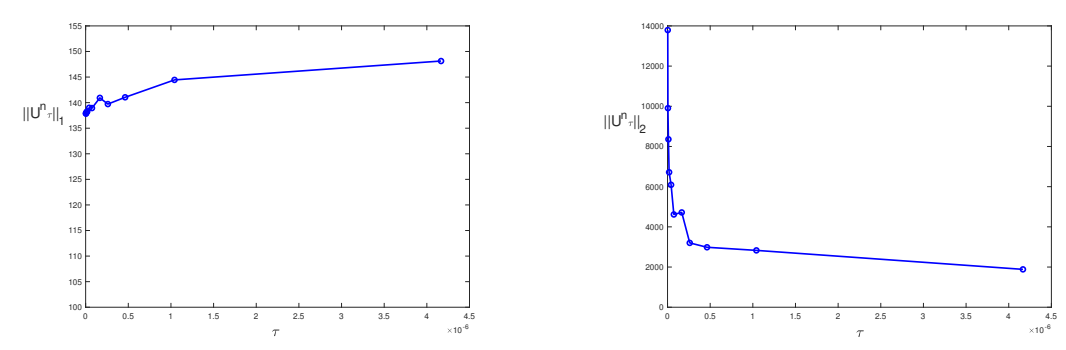

FIG. 4.3. $N=3, \alpha=1, u_{0}(r)=200\left(1-r^{2}\right), H(s)=$ s. Left: $\left\|U^{n_{\tau}}\right\|_{1}$, the discrete $L^{1}$-norm at $T(\tau)$. Right: $\left\|U^{n_{\tau}}\right\|_{2}$, the discrete $L^{2}$-norm at $T(\tau)$.

where the last inequality follows from (4.11). Since $\Delta r=\left(\frac{\tau}{\lambda}\right)^{1 / 2}$, we have, for all $p>\frac{N q}{2}$,

$$
\left\|U^{n_{\tau}}\right\|_{p} \geq\left(\frac{1}{\lambda}\right)^{\frac{N}{2 p}} \tau^{\frac{N}{2 p}-\frac{1}{q}}=\left(\frac{1}{\lambda}\right)^{\frac{N}{2 p}} \tau^{\frac{1}{p q}\left(\frac{N q}{2}-p\right)} \rightarrow \infty \quad \text { as } \quad \tau \rightarrow 0 .
$$

We remark that, for any $q>0,(4.12)$ is satisfied and thus the validity of Theorem 4.13 is guaranteed. Thus, it is interesting to see that different choices of $q$ may lead to different conclusions concerning the blow-up of the $L^{p}$-norms. If we set $q=\alpha$, then we have, for all $p>\frac{N \alpha}{2}$,

$$
\lim _{\tau \rightarrow 0}\left\|U^{n_{\tau}}\right\|_{p}=\infty
$$

which comply with (1.6). If $q<\alpha$, then Theorem 4.14 proves that for $\frac{N q}{2}<p<\frac{N \alpha}{2}$,

$$
\left\|U^{n_{\tau}}\right\|_{p} \rightarrow \infty \quad \text { as } \quad \tau \rightarrow 0
$$

This is not consistent with (1.7), although $\lim _{\tau \rightarrow 0} T(\tau)=T$ is satisfied. We may therefore say that $H(s)=s^{q}$ with $q<\alpha$ is inappropriate. On the other hand, in the case of $q>\alpha$, Theorem 4.14 enables us to infer

$$
\left\|U^{n_{\tau}}\right\|_{p} \rightarrow \infty \quad \text { as } \quad \tau \rightarrow 0
$$

only for $p>\frac{N q}{2}$. To prove the unboundedness of the $L^{p}$-norms in the case of $\frac{N \alpha}{2}<p \leq \frac{N q}{2}$, we need to investigate the behavior of the numerical solution in more detail. The computational results seem to suggest that, for $\frac{N \alpha}{2}<p \leq \frac{N q}{2}$, the $L^{p}$-norms for the numerical solutions still become unbounded at the time step $n_{\tau}$ as $\tau \rightarrow 0$; see Figure 4.4. Nevertheless, the possibility of convergence to a finite value as $\tau \rightarrow 0$ is not excluded theoretically. We do not have any analytical result yet in this case. It is often difficult to tell whether $\left\|U^{n_{\tau}}\right\|_{p}$ remains bounded or tends to infinity as $\tau \rightarrow 0$ for $p<\frac{N \alpha}{2}$; see Figure 4.5. Anyway, we consider the choice of $q=\alpha$ better than others.

REMARK 4.15. In case of $q<\alpha$ and in view of Theorem 4.14, we have, for $\frac{N q}{2}<p<\frac{N \alpha}{2}$,

$$
\left\|U^{n_{\tau}}\right\|_{p} \rightarrow \infty \quad \text { as } \quad \tau \rightarrow 0
$$

while $\|u(t, \cdot)\|_{L^{p}}$ remains bounded as $t \rightarrow T$. This inconsistency is not a serious problem in the following sense. Note that the numerical results suggest that the numerical blow-up time $T(\tau)$ converges from above to the exact blow-up time $T$ if $q<\alpha$ is chosen (see the case of $s^{0.6}$ in Figure 4.2). Note also that Theorem 3.6 guarantees the convergence of the 


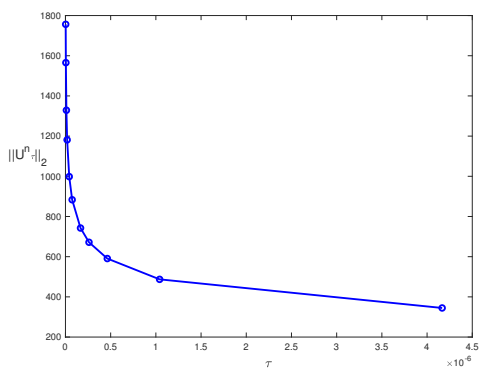

FIG. 4.4. $N=3, \alpha=1, u_{0}(r)=200\left(1-r^{2}\right), H(s)=s^{1.4}$. The discrete $L^{2}$-norms at $T(\tau)$ are plotted against $\tau$. Note that $\frac{N \alpha}{2}<2=p<\frac{N q}{2}$.
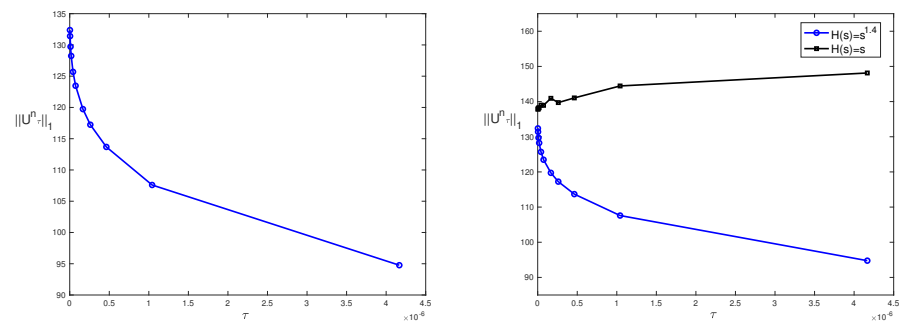

FIG. 4.5. $N=3, \alpha=1, u_{0}(r)=200\left(1-r^{2}\right)$. The discrete $L^{1}$-norms are plotted. Note that $p=1<\frac{N \alpha}{2}$. Left: The result of $H(s)=s^{1.4}$ only. Right: The results of $H(s)=s$ and $H(s)=s^{1.4}$.

numerical solution only up to a time $T_{0}<T$. These results show that (4.14) does not have much significance since $T(\tau)=\tau \cdot n_{\tau}>T>T_{0}$.

To see why a choice of $H$ is important, we introduce the following abstract theorem: Let $u(t)=u(t, \cdot)$ be a solution which blows up in a finite time $T$ of a certain abstract evolution equation. Let $\left\{U^{n}\right\}$ be a numerical solution computed with uniform temporal increment $\tau$ which converges in the following sense: For any $1 \leq p \leq \infty$ and any given $T_{0}<T$,

$$
\lim _{\tau \rightarrow 0} \max _{t_{n} \in\left[0, T_{0}\right]}\left|\left\|U^{n}\right\|_{p}-\left\|u\left(t_{n}, \cdot\right)\right\|_{L^{p}}\right|=0 .
$$

Given any $\tau>0$, assume that there exists a strictly increasing function $H:(0, \infty) \longmapsto(0, \infty)$ satisfying $\lim _{s \rightarrow \infty} H(s)=\infty$ and $n_{\tau} \in \mathbb{N}$, depending on $\tau$ and $H$, such that

$$
\tau \cdot H\left(\left\|U^{n_{\tau}-1}\right\|_{\infty}\right)<1 \quad \text { and } \quad \tau \cdot H\left(\left\|U^{n_{\tau}}\right\|_{\infty}\right) \geq 1 .
$$

We then define the numerical blow-up time $T(\tau)$ by $T(\tau)=\tau \cdot n_{\tau}$ and assume that one can verify the convergence of the numerical blow-up time, namely, $\lim _{\tau \rightarrow 0} T(\tau)=T$. We then have the following theorem:

THEOREM 4.16. Let $1 \leq p<\infty$. Assume that

$$
\limsup _{\tau \rightarrow 0} \max _{0 \leq n \leq n_{\tau}}\left\|U^{n}\right\|_{p}=M<\infty .
$$

Then

$$
\limsup _{t \rightarrow T}\|u(t, \cdot)\|_{L^{p}}<\infty .
$$


Proof. A similar proof can be found in [18]. For the sake of convenience, a sketch of the proof is given. Assume that

$$
\limsup _{t \rightarrow T}\|u(t, \cdot)\|_{p}=\infty .
$$

Then, for all $\varepsilon>0$, there exists $t_{\varepsilon} \in(T-\varepsilon, T)$ such that $2 M<\left\|u\left(t_{\varepsilon}, \cdot\right)\right\|_{p}<\infty$. Thus, one has

$$
\max _{t \in\left[0, \frac{T+t_{\varepsilon}}{2}\right]}\|u(t, \cdot)\|_{p}>2 M
$$

On the other hand, since $\left\{U^{n}\right\}$ is convergent in $\left[0, \frac{t_{\varepsilon}+T}{2}\right]$ and $\lim _{\tau \rightarrow 0} T(\tau)=T$, there exists a sufficiently small $\tau_{\varepsilon}$ such that

$$
T(\tau)>\frac{t_{\varepsilon}+T}{2} \quad \text { and } \quad \max _{k \tau \in\left[0, \frac{t_{\varepsilon}+T}{2}\right]}\left\|U^{k}\right\|_{p}>3 M / 2, \quad \forall \tau \leq \tau_{\varepsilon},
$$

which implies

$$
\max _{k \tau \in[0, T(\tau)]}\left\|U^{k}\right\|_{p}>3 M / 2, \quad \forall \tau \leq \tau_{\varepsilon} .
$$

It then follows that

$$
\limsup _{\tau \rightarrow 0} \max _{0 \leq n \leq n_{\tau}}\left\|U^{n}\right\|_{p} \geq 3 M / 2
$$

which is a contradiction.

Thanks to this theorem, one is able, from the numerical results, to predict the boundedness in the $L^{p}$-norms of the exact solution without knowledge such as (1.7) if $H$ can be suitably chosen. However, since the choice of a suitable $H$ is problem-dependent, a careful analysis should be applied to individual problems.

5. Computation of a minimal blow-up solution. As mentioned before, the blow-up behavior of solutions of (1.2)-(1.5) in higher space dimensions is considerably different from that in lower space dimensions. One difference is the possibility of immediate recovery of regularity after a blow-up. Fila et al. [13] defined the minimal $L^{1}$-solution as follows: By a limit $L^{1}$-solution of $u_{t}=\triangle u+f(u)$, we mean a function $u(t, x)$ which can be approximated by global classical solutions in the following way: There is a sequence $\left\{u_{0, n}\right\}$ in $C(\bar{\Omega})$ such that

$$
u_{0, n} \rightarrow u_{0} \quad \text { in } \quad C(\bar{\Omega})
$$

and that the solution $u_{n}$ with $u_{n}(0, \cdot)=u_{0, n}$ exists in $0 \leq t<\infty$ and satisfies

$$
u_{n}(t, \cdot) \rightarrow u(t, \cdot) \quad \text { in } \quad L^{1}(\Omega)
$$

for every $t>0$, and $f\left(u_{n}\right) \rightarrow f(u)$ in $L^{1}((0, t) \times \Omega)$, for every $t>0$. We refer to any such sequence $\left\{u_{n}\right\}$ as an approximating sequence for $u$. We call $u$ a minimal $L^{1}$-solution if it has an approximating sequence that is pointwise nondecreasing in $n$. Then it is proved in [13] that under certain assumptions the minimal solution, which blows up in finite time, becomes regular immediately after the blow-up time $T$ if $\frac{4}{N-2}<\alpha<\alpha^{*}$, where

$$
\alpha^{*}= \begin{cases}\infty & \text { if } N \leq 10, \\ \frac{4}{N-4-2 \sqrt{N-1}} & \text { if } N>10 .\end{cases}
$$




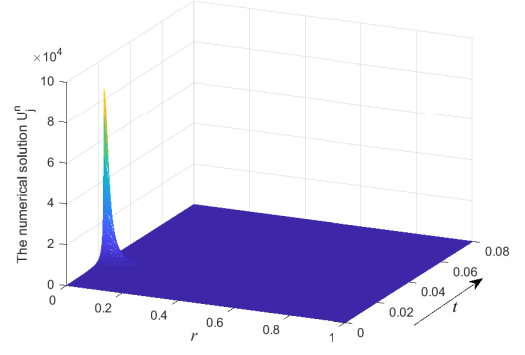

FIG. 5.1. $N=15, u_{0}(r)=A\left(1-r^{2}\right)(A=104.64), J=500, \sigma=\frac{3}{2 N}$. Computation with (3.5)-(3.8) and time increment (3.10).

Later, this result was extended in [19] to all $\alpha>\frac{4}{N-2}$ and non-minimal $L^{1}$-solutions under certain assumption on the blow-up rate. Moreover, the following result was also proved in [19]: Let $\frac{4}{N-2}<\alpha<\infty$, and let $\phi \in H^{1}(\Omega) \cap L^{\infty}(\Omega)$ be nonnegative and not identically zero. Then there exists $\beta^{*}>0$ such that the solution $u^{\beta}$ of (1.2)-(1.5) with initial data $u^{\beta}(0, r)=\beta \phi$ satisfies:

(a) $u^{\beta}$ is globally smooth, and $\left\|u^{\beta}\right\|_{L^{\infty}(\Omega)} \rightarrow 0$ as $t \rightarrow \infty$ if $0 \leq \beta<\beta^{*}$.

(b) $u^{\beta}$ blows up in finite time if $\beta>\beta^{*}$.

(c) $u^{\beta}$ blows up in finite time, and its minimal $L^{1}$-continuation exists for all $t \geq 0$, eventually becomes smooth, and satisfies $\left\|u^{\beta}(t, \cdot)\right\|_{L^{\infty}(\Omega)} \rightarrow 0$ as $t \rightarrow \infty$ if $\beta=\beta^{*}$. See also $[15,20]$.

In view of this result, we now try to compute a minimal blow-up solution as follows. Since the solutions exist globally for small initial data, we set $u_{0}(r)=A\left(1-r^{2}\right)$, for $0 \leq r \leq 1$, and increase the value of $A$ to find out the critical value for the numerical blow-up of $\left\{U_{j}^{n}\right\}$. We carried out an experiment by using (3.5)-(3.8) with $N=15, \alpha=1, J=500$, and $\sigma=\frac{3}{2 N}$. We found that the computation becomes very sensitive near $A=104.64$. In fact, the numerical solution $\left\{U_{j}^{n}\right\}$ remains bounded as $A \leq 104.64$ and blows up for $A=104.65$. Figure 5.1 displays the computational result. The maximum of $\left\{U_{j}^{n}\right\}$ is about 84857.5 and $\left\|U^{n}\right\|_{\infty}$ tends to 0 as $n \rightarrow \infty$.

6. Concluding remarks. In this paper, we considered two numerical schemes for the nonlinear heat equation (1.2)-(1.5). It was revealed that we can reproduce and analyze several blow-up characteristics, such as the blow-up set and the $L^{p}$-norm blow-up, by our numerical methods. However, there are several problems yet to be solved.

Besides immediate regularization after a blow-up, Fila et al. [13] also proved the possibility of multiple blow-ups: Let $u$ be a minimal $L^{1}$-solution of (1.2)-(1.5) which blows up in a finite time $T$. Assume that the initial data $u_{0}(r)$ satisfy $u_{0} \in C([0,1])$ and $u_{0}(r) \geq 0$. Then there exists a positive integer $k$ such that $t_{1}=T<t_{2}<\ldots<t_{k}<\infty$ and $\lim _{t \nearrow t_{i}}\|u(t, \cdot)\|_{L^{\infty}(\Omega)}=\infty$, for all $i=1, \ldots, k$. Moreover, the following estimate holds:

$$
2 k-1 \leq \min _{t \in(0, T)} Z_{[0,1]}\left(u_{t}(t, \cdot)\right),
$$

where $Z_{I}(g)$ denotes the number of zeros of $g$ in $I$. Since we know only a mathematical upper bound of $k$, whether one can determine the number $k$ numerically is an interesting problem.

The blow-up rate is another interesting problem for the numerical analysis. Assume that the solution for (1.2)-(1.5) blows up in a finite time $T$. We say that the blow-up is of Type I if 
the solution $u$ satisfies

$$
\limsup _{t \rightarrow T}(T-t)^{\frac{1}{\alpha}}\|u(t, \cdot)\|_{\infty}<\infty,
$$

while the blow-up is said to be of Type II if $u$ satisfies

$$
\limsup _{t \rightarrow T}(T-t)^{\frac{1}{\alpha}}\|u(t, \cdot)\|_{\infty}=\infty .
$$

It was shown, for example in [14], that the blow-up for (1.2)-(1.5) is always of Type I if

$$
u(0, r) \geq 0 \quad \text { and } \quad u_{t}(0, r) \geq 0, \quad \forall r \in(0,1) .
$$

On the other hand, Mizoguchi [21] proved that a Type II blow-up solution exists if

$$
\alpha>\frac{7}{N-11} \quad \text { and } \quad N \geq 12 .
$$

For more details concerning the theoretical results for Type I and Type II blow-up solutions of (1.2)-(1.5), we refer the readers to [16, 19] and the references therein.

For the computational part of the blow-up rate, Groisman [17] showed that the blow-up for his numerical solution is always of Type I. That is, Type II blow-up cannot be reproduced by his scheme. Later, assuming that the initial data satisfy (6.1), Cho [10] and Lin [18] used the algorithm proposed in [8] to compute a blow-up solution and showed that the blow-up rate for the numerical solution is also of Type I. On the other hand, Anada et al. [2] used the rescaling algorithm of [3] to compute blow-up solutions and their blow-up rates. They showed that Type II blow-up for several model problems can be reproduced by their algorithm. As for the blow-up solutions of (1.2)-(1.5), however, we know no method which can reproduce the Type II blow-up. Numerical analysis for this problem deserves more attention.

Acknowledgments. The first author is supported by the grant 107-2115-M-194-004MY2, Ministry of Science and Technology, Taiwan and is partially supported by the Advanced Institute of Manufacturing with High-tech Innovations (AIM-HI) from The Featured Areas Research Center Program within the framework of the Higher Education Sprout Project by the Ministry of Education in Taiwan. The second author thanks JSPS A3 Foresight Program for various supports. He is also supported by JSPS Kakenhi $18 \mathrm{H} 01137$.

\section{REFERENCES}

[1] L. M. Abia, J. C. LóPEZ-MARcos, AND J. MARTÍNEZ, The Euler method in the numerical integration of reaction-diffusion problems with blow-up, Appl. Numer. Math., 38 (2001), pp. 287-313.

[2] K. ANADA, T. IsHIWATA, AND T. UsHIJIMA, A numerical method of estimating blow-up rates for nonlinear evolution equations by using rescaling algorithm, Jpn. J. Ind. Appl. Math., 35 (2018), pp. 33-47.

[3] M. BERGER AND R. V. KoHN, A rescaling algorithm for the numerical calculation of blowing-up solutions, Comm. Pure Appl. Math., 41 (1988), pp. 841-863.

[4] Y. G. CHEN, Asymptotic behaviours of blowing-up solutions for finite difference analogue of $u_{t}=u_{x x}+$ $u^{1+\alpha}$, J. Fac. Sci. Univ. Tokyo Sect. IA Math., 33 (1986), pp. 541-574.

[5] - Blow-up solutions of a semilinear parabolic equation with the Neumann and Robin boundary conditions, J. Fac. Sci. Univ. Tokyo Sect. IA Math., 37 (1990), pp. 537-574.

[6] - Blow-up solutions to a finite difference analogue of $u_{t}=\Delta u+u^{1+\alpha}$ in N-dimensional balls, Hokkaido Math. J., 21 (1992), pp. 447-474.

[7] X.-Y. Chen And H. Matano, Convergence, asymptotic periodicity, and finite-time blow-up in onedimensional semilinear heat equations, J. Differential Equations, 78 (1989), pp. 160-190.

[8] C.-H. Сно, On the computation of the numerical blow-up time, Jpn. J. Ind. Appl. Math., 30 (2013), pp. 331349. 
[9] - On the finite difference approximation for blow-up solutions of the porous medium equation with a source, Appl. Numer. Math., 65 (2013), pp. 1-26.

[10] C.-H. СHO, A numerical algorithm for blow-up problems revisited, Numer. Algorithms, 75 (2017), pp. 675697.

[11] C.-H. Сho, S. HAmada, AND H. Окамото, On the finite difference approximation for a parabolic blow-up problem, Japan J. Indust. Appl. Math., 24 (2007), pp. 131-160.

[12] C.-H. СHO AND H. OKAмото, Further remarks on asymptotic behavior of the numerical solutions of parabolic blow-up problems, Methods Appl. Anal., 14 (2007), pp. 213-225.

[13] M. FILA, H. MATANo, And P. PolÁčIK, Immediate regularization after blow-up, SIAM J. Math. Anal., 37 (2005), pp. 752-776.

[14] A. FRIEDMAN AND B. MCLEOD, Blow-up of positive solutions of semilinear heat equations, Indiana Univ. Math. J., 34 (1985), pp. 425-447.

[15] V. Galaktionov AND J. L. VÁZQUeZ, Continuation of blow-up solutions of nonlinear heat equations in several space dimensions, Comm. Pure Appl. Math., 50 (1997), pp. 1-67.

[16] Y. GigA AND R. V. KoHn, Characterizing blowup using similarity variables, Indiana Univ. Math. J., 36 (1987), pp. 1-40.

[17] P. GROISMAN, Totally discrete explicit and semi-implicit Euler methods for a blow-up problem in several space dimensioins, Computing, 76 (2006), pp. 325-352.

[18] S.-H. Lin, Numerical Blow-up Behavior for Axisymmetric Solutions of Semilinear Heat Equation, Master Thesis (in Chinese), National Chung Cheng University, Taiwan, 2018. (S.-H. Lin got her Master degree at National Chung Cheng University under the guidance of the first author.)

[19] H. MATANO AND F. MERLE, Classification of type I and type II behaviors for a supercritical nonlinear heat equation, J. Funct. Anal., 256 (2009), pp. 992-1064.

[20] N. Mizoguchi, Type-II blowup for a semilinear heat equation, Adv. Differential Equations, 9 (2004), pp. 1279-1316.

[21] - Boundedness of global solutions for a supercritical semilinear heat equation and its application, Indiana Univ. Math. J., 54 (2005), pp. 1047-1059.

[22] - Various behaviors of solutions for a semilinear heat equation after blowup, J. Func. Anal., 220 (2005), pp. 214-227.

[23] - Multiple blowup of solutions for a semilinear heat equation, Math. Ann., 331 (2005), pp. 461-473.

[24] - On the uniqueness of $L^{1}$-continuation after blowup, J. Funct. Anal., 254 (2008), pp. 2893-2910.

[25] C. E. MUELleR AND F. B. WeISSLER, Single point blow-up for a general semilinear heat equation, Indiana Univ. Math. J., 34 (1985), pp. 881-913.

[26] T. NAKAGAWA, Blowing up of a finite difference solution to $u_{t}=u_{x x}+u^{2}$, Appl. Math. Optim., 2 (1975), pp. 337-350.

[27] T. NAKANISHI AND N. SAITO, Finite element method for radially symmetric solution of a multidimensional semilinear heat equation, Jpn. J. Ind. Appl. Math., 37 (2020), pp. 165-191.

[28] W. REN AND X.-P. WANG, An iterative grid redistribution method for singular problems in multiple dimensions, J. Comput. Phys., 159 (2000), pp. 246-273.

[29] A. M. Stuart And M. S. Floater, On the computation of blow-up, European J. Appl. Math., 1 (1990), pp. 47-71.

[30] F. WEISSLER, $L^{p}$-energy and blow-up for a semilinear heat equation, in Nonlinear Functional Analysis and its Applications. Part 2, F. E. Browder, ed., Proc. Symp. Pure Math., 45, AMS, Providence, 1986, pp. 545-551. 